\title{
Gap Junction-Mediated Signaling from Motor Neurons Regulates Motor Generation in the Central Circuits of Larval Drosophila
}

\author{
Teruyuki Matsunaga , ${ }^{\circledR}$ Hiroshi Kohsaka, ${ }^{1,2}$ and ${ }^{\circledR A}$ Akinao Nose ${ }^{1,2}$ \\ 'Department of Complexity Science and Engineering, University of Tokyo, Chiba 277-8561, Japan and 2Department of Physics, Graduate School of Science, \\ University of Tokyo, Tokyo 113-0033, Japan
}

In this study, we used the peristaltic crawling of Drosophila larvae as a model to study how motor patterns are regulated by central circuits. We built an experimental system that allows simultaneous application of optogenetics and calcium imaging to the isolated ventral nerve cord (VNC). We then investigated the effects of manipulating local activity of motor neurons (MNs) on fictive locomotion observed as waves of MN activity propagating along neuromeres. Optical inhibition of MNs with halorhodopsin3 in a middle segment (A4, A5, or A6), but not other segments, dramatically decreased the frequency of the motor waves. Conversely, local activation of MNs with channelrhodopsin2 in a posterior segment (A6 or A7) increased the frequency of the motor waves. Since peripheral nerves mediating sensory feedback were severed in the VNC preparation, these results indicate that MNs send signals to the central circuits to regulate motor pattern generation. Our results also indicate segmental specificity in the roles of MNs in motor control. The effects of the local MN activity manipulation were lost in shaking- $B^{2}\left(\operatorname{shakB} B^{2}\right)$ or ogre ${ }^{2}$, gap-junction mutations in Drosophila, or upon acute application of the gap junction blocker carbenoxolone, implicating electrical synapses in the signaling from MNs. Cell-type-specific RNAi suggested shakB and ogre function in MNs and interneurons, respectively, during the signaling. Our results not only reveal an unexpected role for MNs in motor pattern regulation, but also introduce a powerful experimental system that enables examination of the input- output relationship among the component neurons in this system.

Key words: calcium imaging; Drosophila; gap junctions; motoneurons; optogenetics; shakB

Significance Statement

Motor neurons are generally considered passive players in motor pattern generation, simply relaying information from upstream interneuronal circuits to the target muscles. This study shows instead that MNs play active roles in the control of motor generation by conveying information via gap junctions to the central pattern-generating circuits in larval Drosophila, providing novel insights into motor circuit control. The experimental system introduced in this study also presents a new approach for studying intersegmentally coordinated locomotion. Unlike traditional electrophysiology methods, this system enables the simultaneous recording and manipulation of populations of neurons that are genetically specified and span multiple segments.

\section{Introduction}

Animal movement is accomplished by spatially and temporally coordinated contraction of various muscles throughout the body

\footnotetext{
Received May 3, 2016; revised Dec. 7, 2016; accepted Jan. 9, 2017.

Author contributions: T.M., H.K., and A.N. designed research; T.M. performed research; T.M. contributed unpublished reagents/analytic tools; T.M. analyzed data; T.M., H.K., and A.N. wrote the paper.

This work was supported by Ministry of Education, Culture, Sports, Science and Technology/Ministry of Education, Culture, Sports, Science and Technology (JSPS) KAKENHI Grants 22115002, 22150003, and 15H04255 to A.N. and 15J03655 to H.K., and JSPS Research Fellowships for Young Scientists to T.M. We are grateful to Drs. Corey S. Goodman, Loren Looger, Vivian Budnik, Leslie Griffith, Robert J. Wyman, Tzumin Lee, and the Bloomington and Kyoto Stock Centers for fly stocks and reagents; Albert Cardona and his colleagues for the use of their EM reconstruction data; and Stefan Pulver and Leslie Griffith for technical advice.

The authors declare no competing financial interests.

Correspondence should be addressed to Akinao Nose, 5-1-5 Kashiwanoha, Kashiwa-shi, Chiba, 277-8561, Japan. E-mail: nose@neuro.k.u-tokyo.ac.jp.
}

(Marder and Calabrese, 1996; Grillner, 2003; Mulloney and Smarandache-Wellmann, 2012). It is generally thought that a neuronal network composed of premotor interneurons generates a motor pattern, and this network sequentially activates different classes of motor neurons (MNs). In this view, MNs play only passive roles in pattern generation, relaying the information they receive from upstream interneuronal networks to muscles. By contrast, there is some evidence that MNs themselves contribute to the motor pattern generation. In the crustacean stomatogastric ganglion and in leech swimming circuits, MNs are part of the pattern-forming network (Brodfuehrer et al., 1995; Marder and 
Calabrese, 1996). In mammalian spinal cords, MNs send a collateral to innervate Renshaw cells, which in turn convey feedback signals to MNs (Moore et al., 2015). However, whether and how MNs regulate motor pattern generation during animal movements remains largely unexplored.

Larval Drosophila is emerging as an excellent model system for studying motor pattern generation since one can apply powerful genetic tools including a large collection of Gal4-drivers (Manning et al., 2012; Li et al., 2014) to study the function of individual component neurons in a numerically simple nervous system (Heckscher et al., 2012, 2015; Kohsaka et al., 2012, 2014; Vogelstein et al., 2014; Couton et al., 2015). Furthermore, previous development of a platform for electronic microscope (EM) image data reconstruction of the entire nervous system of the larval CNS now allows mapping of the circuit structure that mediates specific behaviors (Heckscher et al., 2015; Ohyama et al., 2015; Fushiki et al., 2016; Schneider-Mizell et al., 2016). The larval ventral nerve cord (VNC) consists of three thoracic neuromeres (T1, T2, and T3) and eight abdominal neuromeres (A1-A8). Larval peristaltic crawling is accomplished by successive bilateral muscle contraction that propagates from tail to head (Fox et al., 2006; Heckscher et al., 2012). Muscle contraction in each segment is in turn regulated by sequential activation of MNs in the corresponding neuromere of the VNC (Pulver et al., 2015). Although recent studies have begun to identify several types of premotor interneurons that regulate aspects of movement such as the speed of locomotion and left-right or intersegmental coordination (Kohsaka et al., 2014; Heckscher et al., 2015; Tastekin et al., 2015; Fushiki et al., 2016), how a motor pattern is generated by the neural circuits remains largely unknown.

In a previous study, we used halorhodopsin ( $\mathrm{NpHR}$ ) to locally and transiently inhibit MN activity in one or a few segments and found that local activity perturbation halts the propagation of the peristaltic wave at the site of manipulation (Inada et al., 2011). This suggests that MNs are part of the neural circuits that generate the peristaltic wave. However, how information is retrogradely transmitted from MNs to the central circuits remained unknown. Furthermore, since muscle contraction was used as a measure of the motor outputs, changes in the activity dynamics in the CNS could not be studied. We extended that study by constructing a new experimental system in which we could study the effects of local optogenetic manipulation of MNs on global motor activity in the VNC. We found that optical inhibition of MNs in a middle segment (A4, A5, or A6) decreased the motor frequency. Conversely, photoactivation of MNs in a posterior segment (A6 or A7) increased the frequency of the motor wave. These results indicate that the local activity level of MNs impacts the global outputs of the motor circuits in a segment-specific manner. We also show that gap junctions are involved in this process. While this manuscript was in preparation, a study in zebrafish reported that motor neurons retrogradely influence the activity level of the premotor V2a interneurons via gap junctions and regulate motor generation (Song et al., 2016). Thus, regulation by gap junction-mediated retrograde $\mathrm{MN}$ signaling appears to be a common mechanism of motor control.

\section{Materials and Methods}

Isolation of the nerve cord. Calcium imaging and optogenetic manipulations were conducted in isolated VNCs prepared as described below. Third instar larvae of either sex were pinned down dorsal side up on sylgard-coated Petri dishes. An incision along the dorsal body was made with fine scissors and the internal organs were removed. The body wall was pinned flat. The CNS (including the brain, subesophageal ganglion, and VNC) was dissected away from the body wall and placed dorsal side up on double-sided tape on a glass slide. The two hemispheres of the brain were then cut away from the VNC with microscissors (MB-50-7, Natsume Seisakusho). All dissections and experiments were conducted in physiological saline containing the following (in mм): $135 \mathrm{NaCl}, 5 \mathrm{KCl}, 2 \mathrm{CaCl}_{2}, 4 \mathrm{MgCl}_{2}, 52$-[[1,3dihydroxy-2-(hydroxymethyl)propan-2-yl] amino] ethanesulfonic acid, and 36 sucrose (Marley and Baines, 2011).

Fly stocks. Flies were reared on cornmeal-based food at room temperature $\left(25^{\circ} \mathrm{C}\right)$. We used the GAL4/UAS system (Brand and Perrimon, 1993 ) to drive expression of the calcium indicators GCaMP6m (Chen et al., 2013) and RCaMP (Akerboom et al., 2013), yellow light-sensitive $\mathrm{Cl}^{-}$ion pump NpHR3::mCherry, and blue light-sensitive cation channel channelrhodopsin2 (ChR2) (T159C)::YFP. OK6-GAL4 (Aberle et al., 2002), OK371 (Mahr and Aberle, 2006), and C380-GAL4; Cha-GAL80 (Koh et al., 1999) were used as MN drivers (Sanyal, 2009). We used aCC and RP2 motoneurons (RRA)-Gal4-F (Fujioka et al., 2003) and CQ2Gal4 (Landgraf et al., 2003) to express NpHR3::mCherry or ChR2 (T159C)::YFP in small subsets of MNs and examined the effects of the photostimulation by calcium imaging with RGECO1 (lexAop2-RGECO1; Kohska et al., 2014) and GCaMP6f (13XLexAop2-IVSGCaMP6f-p10; obtained from the Bloomington Stock Center; D. Kim, unpublished data), respectively, whose expression driven by the $t u b$ LexA driver (Lai and Lee, 2006). The alleles of shaking-B (shakB) and ogre, shakB ${ }^{2}$ (Phelan et al. 2008) and ogre $e^{2}$ (Curtin et al., 2002), were used. For RNAi knockdown of shakB and ogre, shakB-RNAi (57706: Valium 20) and ogre-RNAi (stock no. 44048, Valium 20; Pézier et al., 2016) were used.

Chemicals. Carbenoxolone disodium salt (CBX), a gap junction antagonist, was used at a concentration of $1 \mathrm{~mm}$ (Blankenship and Feller, 2010; C4790-1G, Sigma).

Calcium imaging. A genetically encoded calcium indicator, GCaMP6m (Chen et al., 2013) or RCaMP (Akerboom et al., 2013), was used for calcium imaging. Imaging was conducted on the isolated VNCs prepared as described above. Images were acquired by an EMCCD camera (iXon) connected to confocal microscopy (FV1000, Olympus) via a C-mount attachment $(1 \times$ magnification $)$. For detection of GCaMP6m signals, uniform blue light filtered by a $470-495$ $\mathrm{nm}$ filter from a mercury lamp source was applied to the preparation, and the fluorescence signal was detected by EMCCD camera through a standard GFP filter unit (U-MNIBA3, Olympus). For detection of RCaMP signals, green light filtered by a $545-580 \mathrm{~nm}$ filter from a mercury lamp was applied, and the signals were detected through a $610 \mathrm{~nm}$ high-pass filter.

A water-immersion $40 \times$ objective lens (numerical aperture, 0.8 ; Achroplan, Zeiss), which allows imaging of most of the neuromeres in the VNC, was used. We acquired 1800-3000 frames of images in 10 or 5 frames/s. For time course analysis, we defined regions of interest (ROIs) in preparation and plotted the mean fluorescent intensity in the ROIs of each image using ImageJ software (NIH).

Optogenetics. Photostimulation was applied using standard upright confocal microscopy (FV1000, Olympus; Matsunaga et al., 2013). For stimulation of NpHR3, yellow light $\left(100 \mathrm{~mW} / \mathrm{mm}^{2}, 559 \mathrm{~nm}\right)$ derived from a semiconductor laser and filtered through a $559 \mathrm{~nm}$ dichroic mirror was used. For stimulation of ChR2, green light $\left(6 \mathrm{~mW} / \mathrm{mm}^{2}\right.$, $488 \mathrm{~nm}$ ) derived from an argon laser and filtered through a $488 \mathrm{~nm}$ dichroic mirror was used. Spatial and temporal control of the photostimulation was achieved by a galvanometer mirror. Since the spot size of the laser equipped with the confocal microscope was too small $(<1 \mu \mathrm{m})$ to activate a group of neurons, laser light was defocused to the size of a hemisegment $(\sim 30 \mu \mathrm{m})$ by incorporation of a pinhole (2000 $\mu \mathrm{m}$ diameter) and a convex lens in the light pathway (Olympus). Photostimulation was applied for 30 or $60 \mathrm{~s}$. For simultaneous photostimulation and calcium imaging, light for imaging was applied through an independent light pathway, and the fluorescent signal was detected by an EMCCD camera attached to the confocal microscope as described above. 

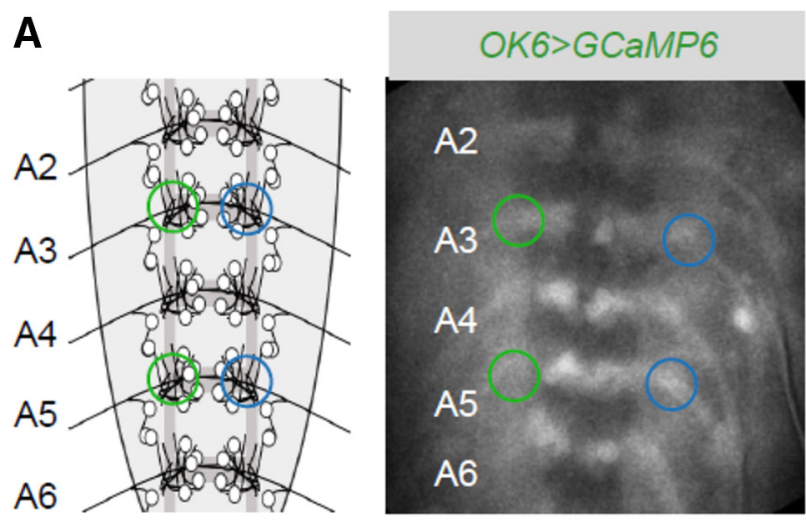

B

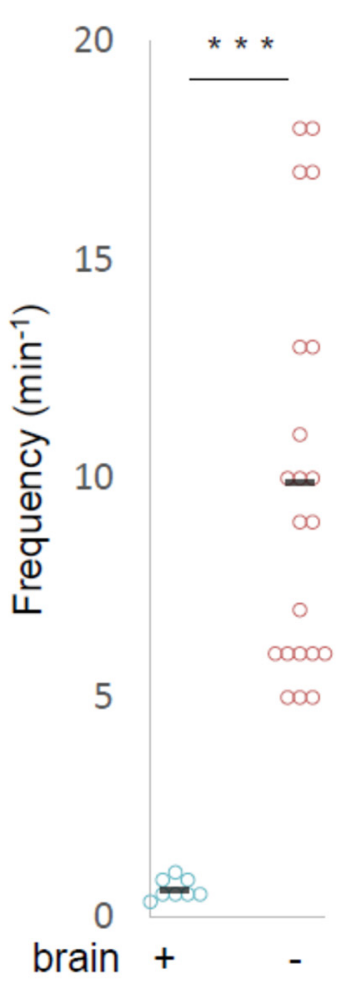

C

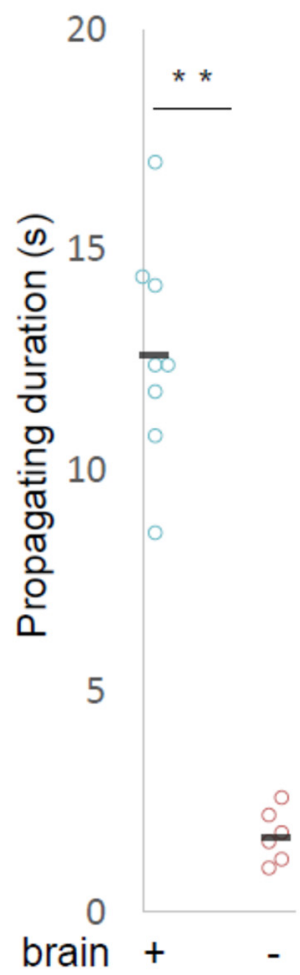

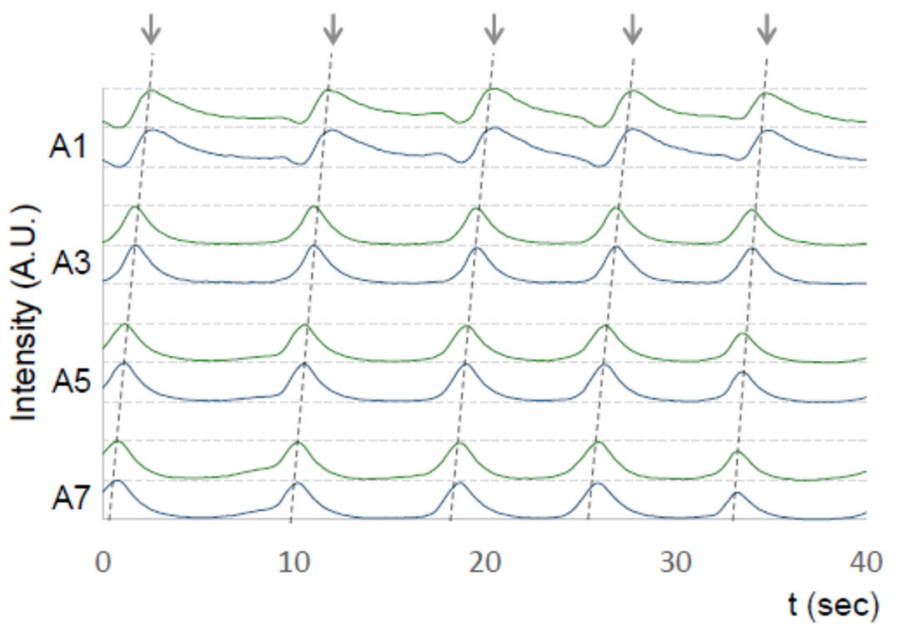

D

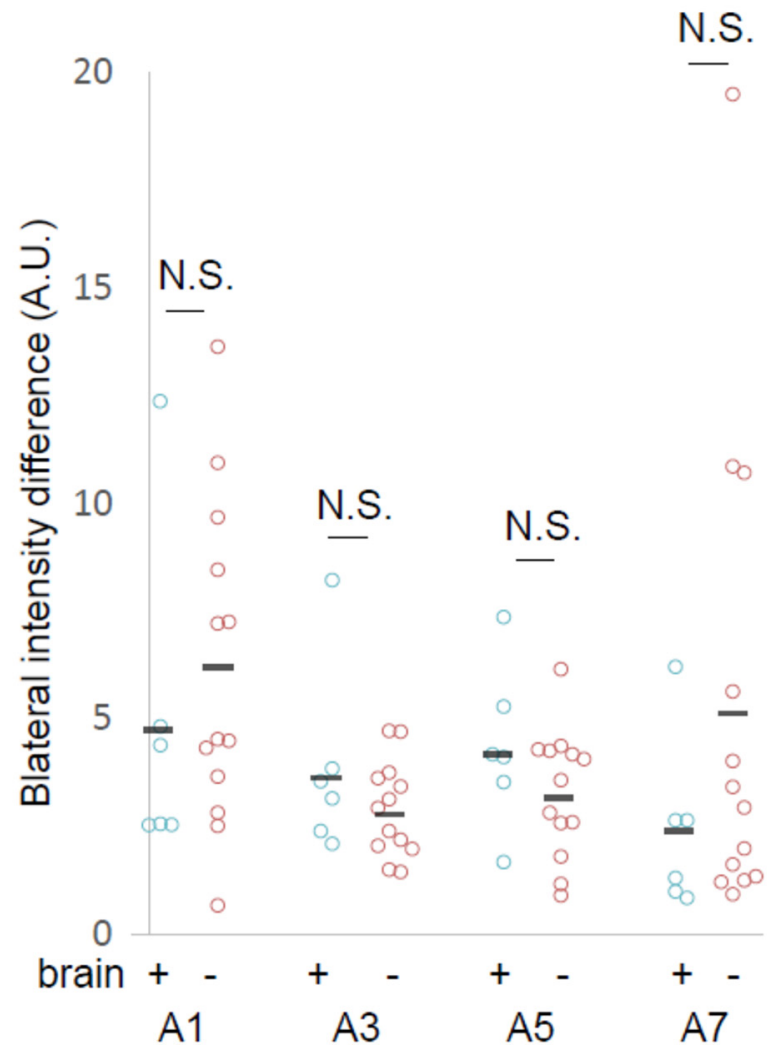

Figure 1. Increased forward motor waves in the absence of the brain. $A$, Calcium imaging of MNs in an isolated CNS without the brain. The schematic (left) and photograph (middle) show ROls used for the study (green and blue circles). Representative plots of the fluorescent changes in $A 1, A 3, A 5$, and $A 7$ segments are shown. Fluorescent intensity in the left and right sides of each segment is shown in green and blue, respectively. Dashed lines indicate forward motor waves. $\boldsymbol{B}-\boldsymbol{D}$, Frequency $(\boldsymbol{B})$, propagating duration $(A 7$ to $A 1 ; \boldsymbol{C})$, and normalized bilateral intensity difference $(\boldsymbol{D})$ of motor waves in isolated CNS with or without the brain are compared. Black bars in $\boldsymbol{B}-\boldsymbol{D}$ and in subsequent figures indicate the average. ${ }^{* *} p<0.01$; ${ }^{* * *} p<0.001$ (Mann-Whitney U test).

Immunohistochemistry. For immunostaining, dissected larvae were fixed in $4 \%$ formaldehyde in PBS at room temperature for $30 \mathrm{~min}$, washed repeatedly with PBS, placed in PBS with $0.2 \%$ Triton X-100 $(\mathrm{PBT})$, in PBT with normal goat serum, and incubated with primary antibodies overnight at $4^{\circ} \mathrm{C}$. After rinses, secondary antibodies were applied. Primary antibodies used in this study were as follows: rabbit anti-GFP (Frontier Science, Af2020, 1:1000), mouse anti-Fasciclin2 (Developmental Studies Hybridoma Bank, 1D4, 1:10), and rabbit antiDsRed (Clontech, catalog \#632496, 1:500). Secondary antibodies used were Alexa488-conjugated goat anti-rabbit IgG (Invitrogen, 1:300) and Alexa55-conjugated goat anti-mouse IgG (Invitrogen). For imaging and analysis, a confocal laser-scanning microscope and its software (Flu- oview 1000, Olympus) with a water-immersion $60 \times$ objective lens (Olympus) were used.

Data analysis. The intensity of the calcium imaging signal was normalized using the difference between maximum and minimum intensities in all frames or the amplitude of the wave. Occurrence of motor waves was defined as the existence of sequential elevation of calcium signals in at least three successive neuromeres. Frequency was calculated based on the number of calcium waves in the $30 \mathrm{~s}$ period just before, during, and just after the photostimulation. The propagation duration was defined as the time difference between the peaks of fluorescent intensity in two segments of interest. Normalized bilateral intensity difference was defined as the average of intensity difference between the left and right ROIs during a motor wave. For example, a normalized 
A

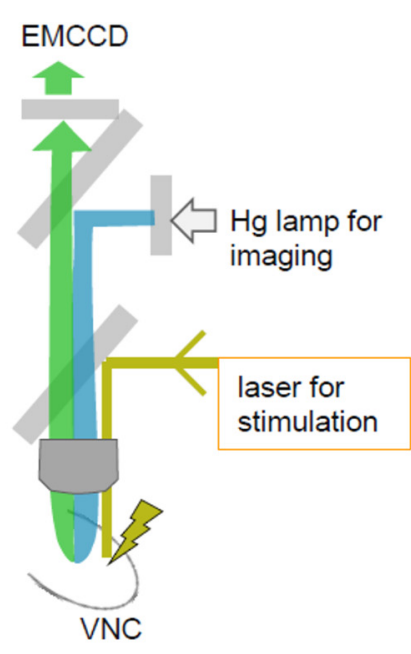

C
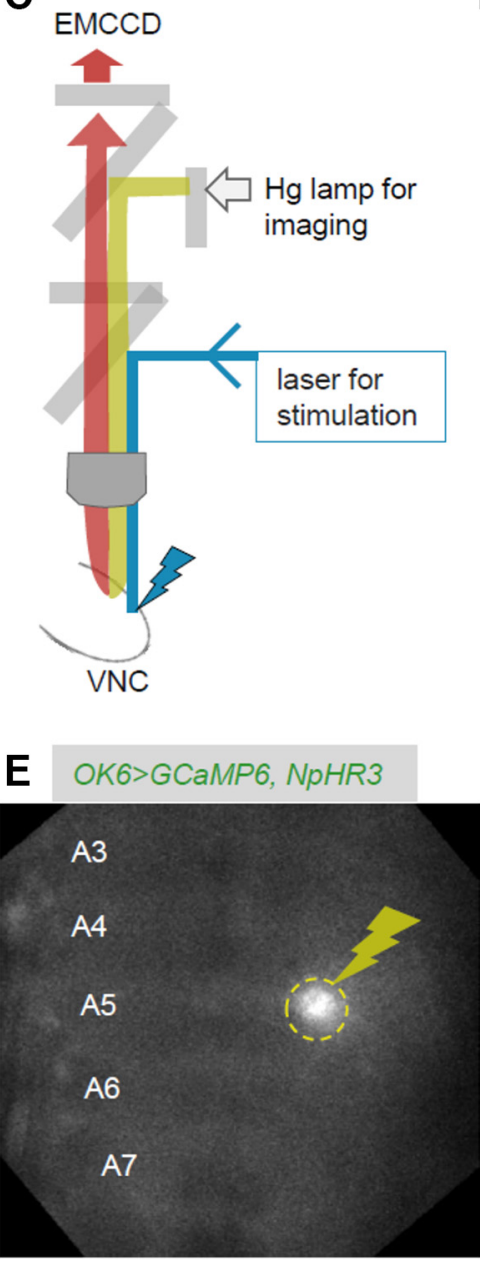

B

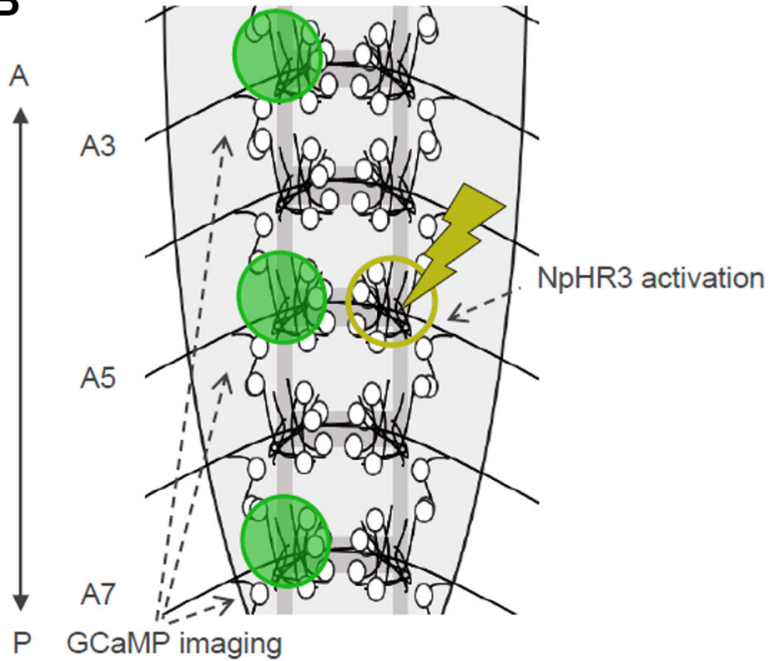

D

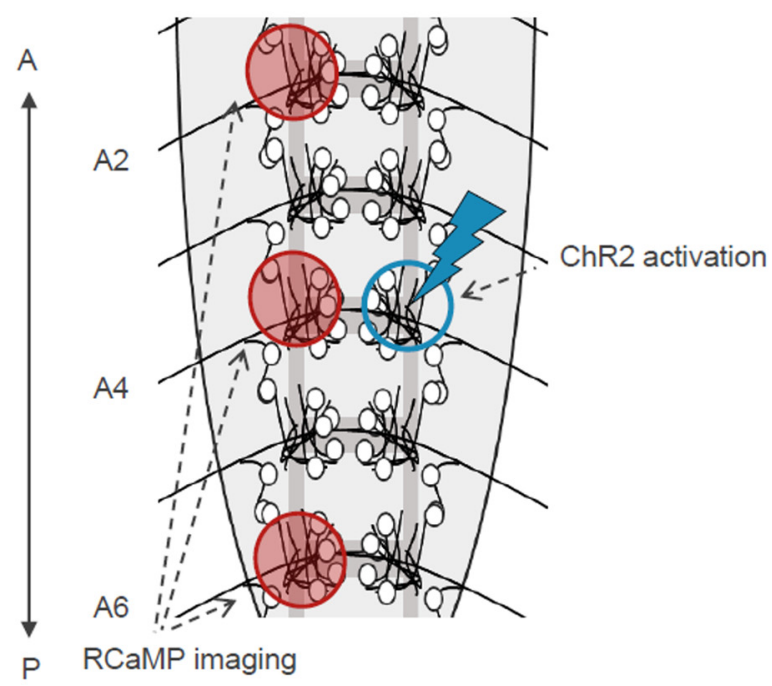

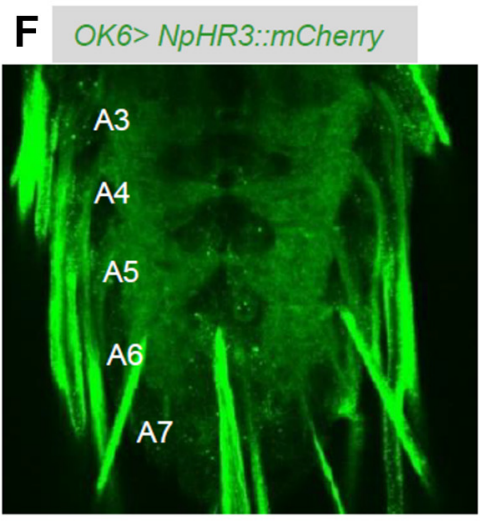

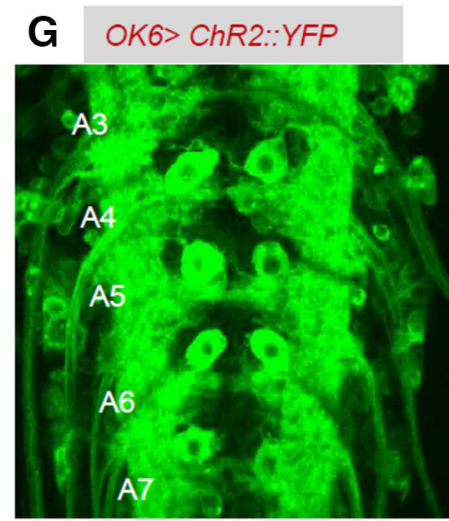

Figure 2. The experimental system used for simultaneous local photomanipulation and calcium imaging. $\boldsymbol{A}, \boldsymbol{B}$, Schematics showing the experimental setup $(\boldsymbol{A})$ and ROls $(\boldsymbol{B})$ for simultaneous photoinhibition and calcium imaging. $\boldsymbol{C}, \boldsymbol{D}$, Schematics showing the experimental setup $(\boldsymbol{C})$ and $R O$ Is $(\boldsymbol{D})$ for simultaneous photoactivation and calcium imaging. $\boldsymbol{E}$, Snapshot during the experiment showing the light stimulation for optical inhibition (dashed yellow circle). $\boldsymbol{F}, \mathbf{G}$, NpHR3::mCherry $(\boldsymbol{F})$ and UAS-ChR2 (T159C)::YFP (G) expression in MNs. A, Anterior; P, posterior.

bilateral intensity difference of A4 $=\Sigma\left|I_{\mathrm{A} 4 \text { left } k}-I_{\mathrm{A} 4 \text { right } k}\right| /$ number of all frames, where $k$ is defined as a frame number during a motor wave. Baseline intensity was normalized to the baseline intensity of the ROI just before the application of photostimulation.

EM reconstruction. Serial section transmission electron microscopy (ssTEM) data were analyzed as described previously (Ohyama et al.,
2015). We searched for and identified membrane contacts between a pair of MNs reconstructed by Zwart et al. (2016).

Statistics. For pairwise comparisons, Wilcoxon signed-rank test was used. The Mann-Whitney $U$ test was applied to compare the difference between two independent groups. For comparison of multiple segments, Steel's test was used. The detection of peak calcium signal was accomplished using Microsoft Excel. 
A

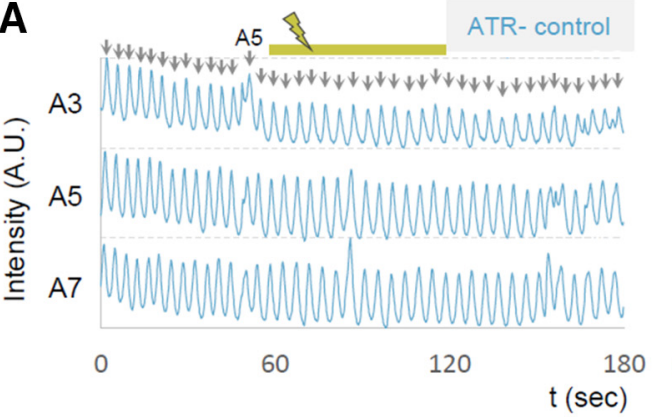

B
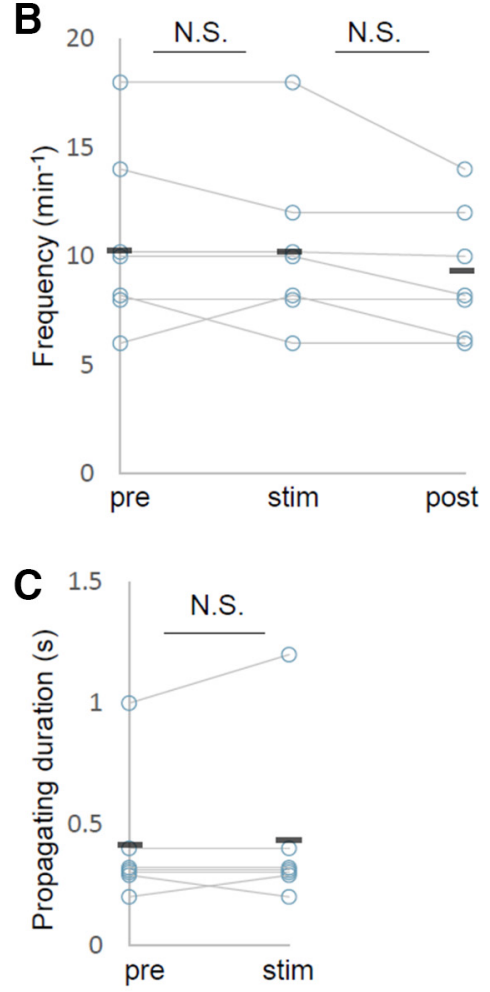

D

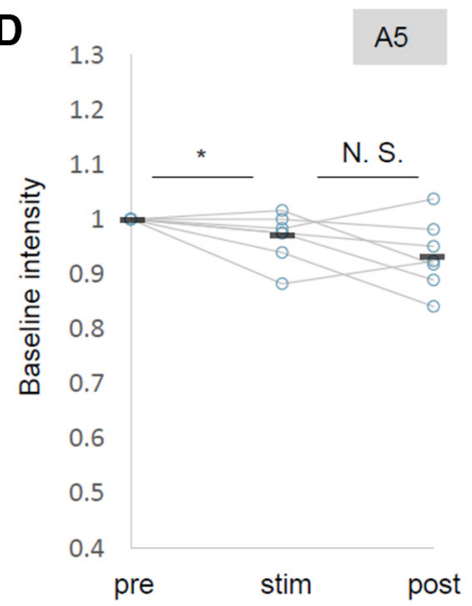

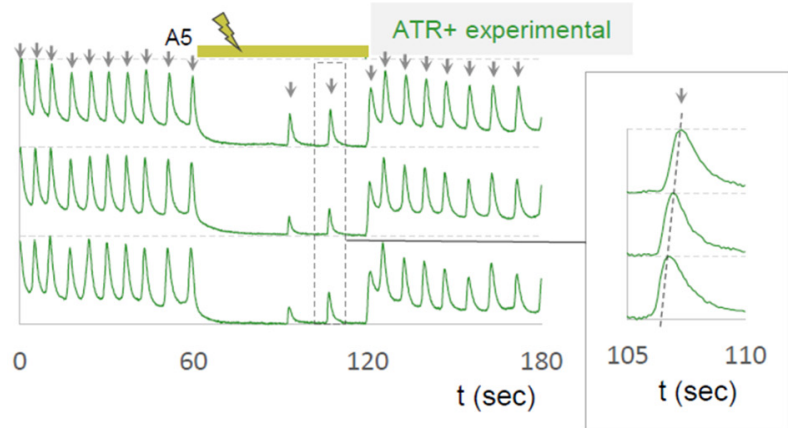

$\mathbf{E}$
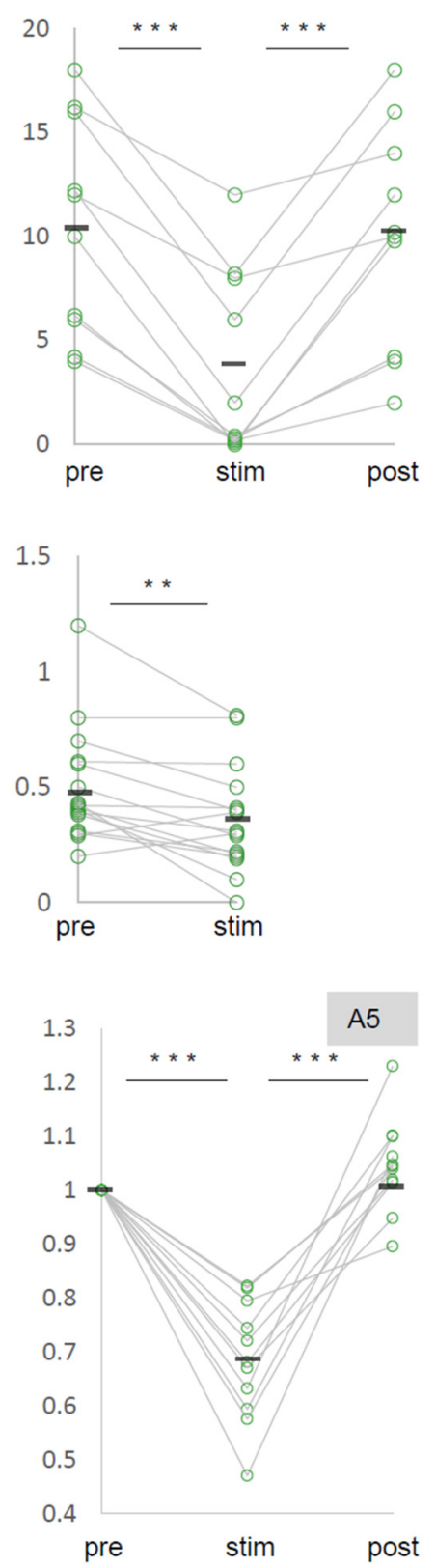

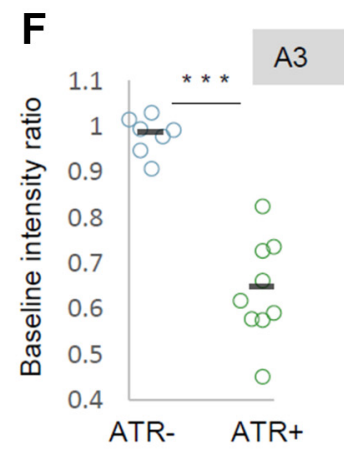

G
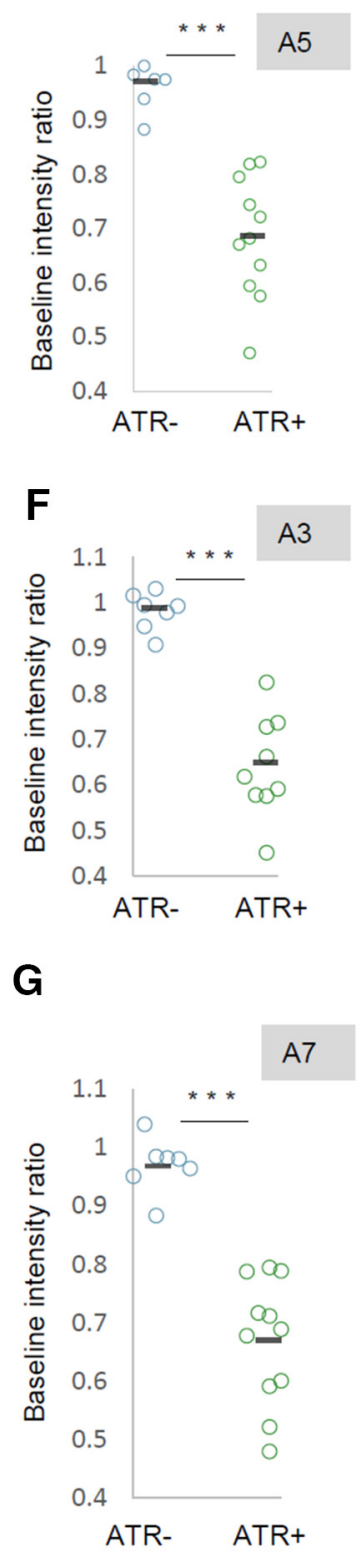

Figure 3. Local photoinhibition of MNs in the A5 segment dramatically reduces the frequency of motor waves. MN activity was analyzed by calcium imaging before (pre), during (stim), and after (post) the optical inhibition of MNs in A5. OK6-Gal 4 was used to express GCaMP6m and UAS-NpHR3::mCherry in MNs. A, Representative plots of normalized fluorescent intensity in control (ATR ${ }^{-}$, left) and experimental (ATR ${ }^{+}$, right) groups. ROls were made in a neuropile region surrounding the nerve root of $A 3, A 5$, and A7 in the side contralateral to the optical manipulation, and in the manipulated side of $A 5$ as shown in Figure $2 B$. Yellow bars $(60-120 \mathrm{~s})$ indicate the duration of light stimulation. Enlarged plots in the dashed rectangle are shown in the inset. $\boldsymbol{B}-\boldsymbol{D}$, Changes in the frequency of motor waves $(\boldsymbol{B})$, propagating duration (A6 to $A 4 ; \boldsymbol{C}$ ), and normalized baseline intensity in $\mathrm{A5}$ (D) upon optical manipulation are plotted. $\boldsymbol{E}-\boldsymbol{G}$, Changes in baseline intensity in $\mathrm{A} 5(\boldsymbol{E}), \mathrm{A} 3(\boldsymbol{F})$, and $\mathrm{A} 7(\boldsymbol{G}) .{ }^{*} p<0.05 ;{ }^{* *} p<0.01 ;{ }^{* * *} p<0.001$ (Wilcoxon signed-rank test; $\boldsymbol{B}-\boldsymbol{D}$ ); ${ }^{* * *} p<0.001$ (Mann-Whitney $U$ test; $\boldsymbol{E}-\boldsymbol{G}$ ). 


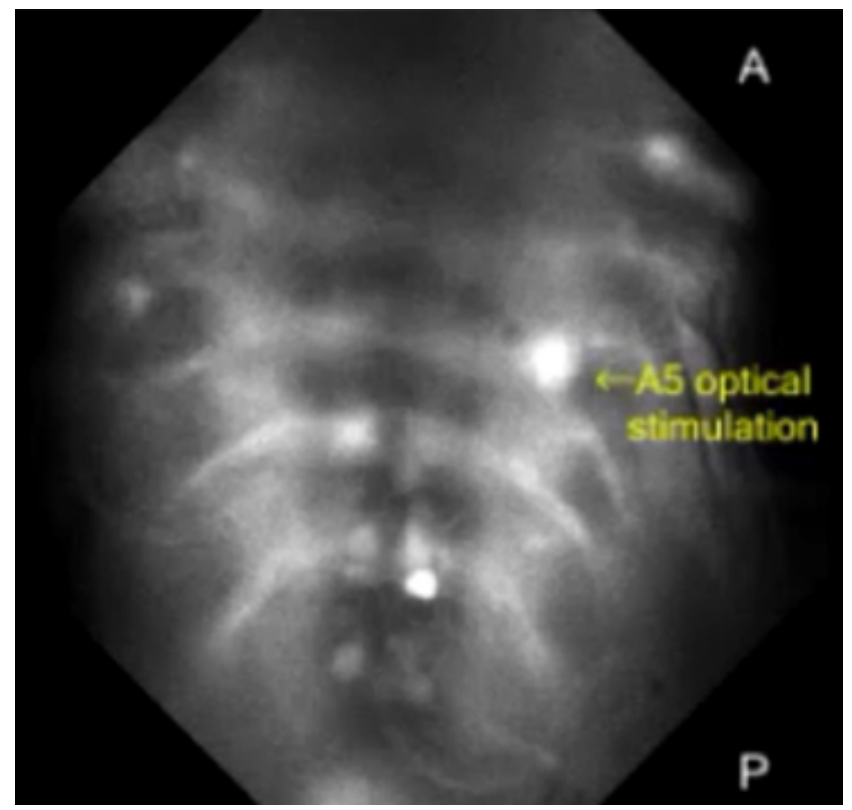

Movie 1. The effects of local photoinhibition of A5 MNs in a control $\left(\right.$ ATR $^{-}$) larva. This movie is related to Figure 3 . The speed of the movie is $4 \times$ the speed of the raw data.
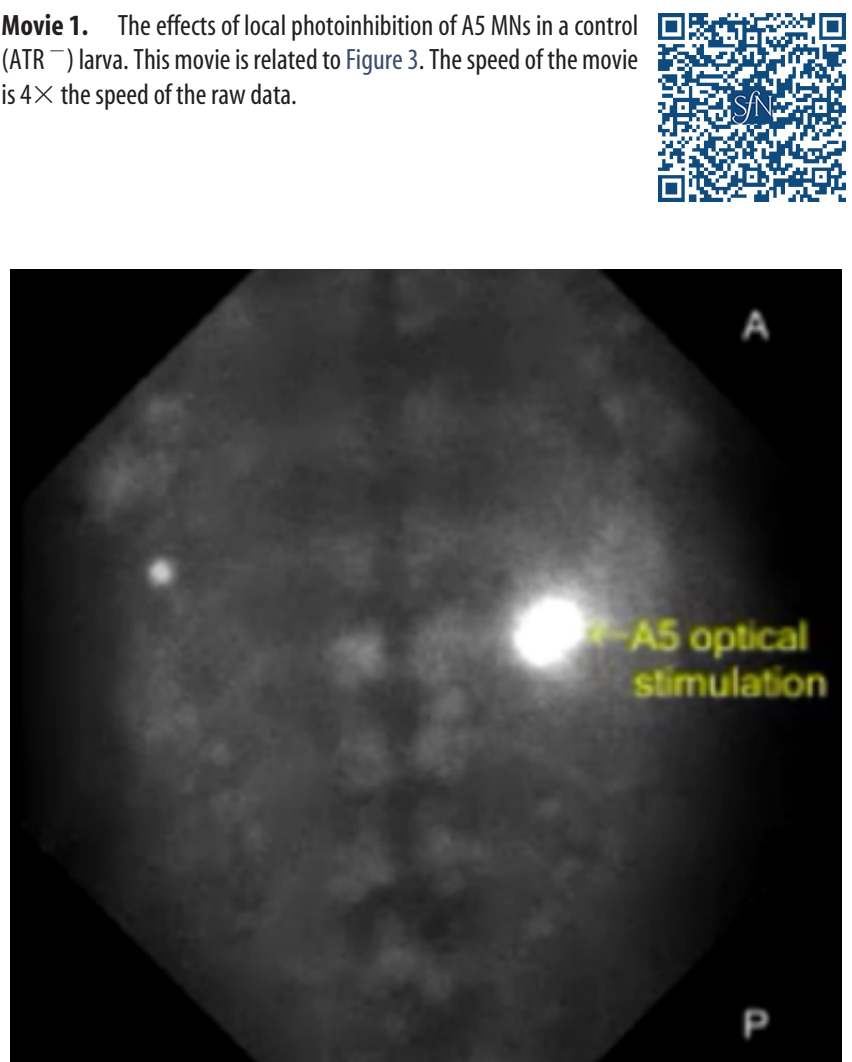

Movie 2. The effects of local photoinhibition of A5 MNs in an experimental $\left(\mathrm{ATR}^{+}\right.$) larva. This movie is related to Figure 3. The speed of the movie is $4 \times$ the speed of the raw data.

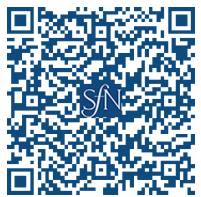

\section{Results}

Efficient imaging of forward motor activity in isolated VNCs Larval peristaltic crawling is accomplished by successive bilateral muscle contractions that propagate from the tail to head. Since muscle contraction in each segment is achieved by activation of MNs in

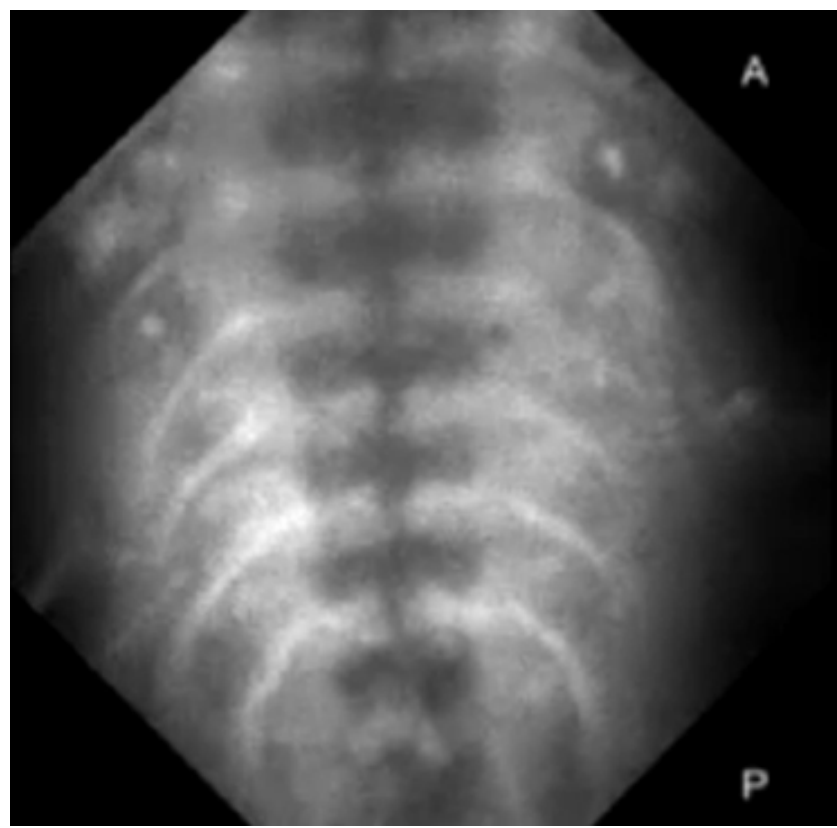

Movie 3. Resumption of motor waves after termination of the local photoinhibition. This movie is related to Figure 3 . The speed of the movie is $4 \times$ the speed of the raw data.

the corresponding neuromere (Fox et al., 2006; Heckscher et al., 2012), the peristaltic locomotion coincides with a wave of MN activity that propagates along the segments (Kohsaka et al., 2014). The wave-like activity of MNs (hereafter called motor waves) can be observed in the isolated CNS using calcium imaging (fictive locomotion; Lemon et al., 2015; Pulver et al., 2015). However, the frequency of the motor waves seen in the isolated CNS is much less $(\sim 1$ event/ 100 s) compared to that of forward peristalsis in the intact larvae $(\sim 1$ event/1 s; Lemon et al., 2015). Since we wanted to systematically study the effects of local activity manipulation of MNs on motor waves, we first tried to develop an experimental system in which we could record motor waves in a more robust manner. We found that removing the brain increased the frequency of motor waves, so we used this preparation (VNC only) for the experiments described below. Figure $1 A$ shows the motor activity recorded in the isolated CNS without the brain. Activities corresponding to motor waves were detected as peaks of fluorescent intensity propagating from the posterior to anterior neuromeres. The waves occur more frequently in the absence of the brain than in the intact CNS [9.9 $\pm 0.99(n=$ $21)$ vs $0.6 \pm 0.08(n=8)$ waves per $\min$ in the absence and presence of the brain, respectively; Fig. $1 B$ ]. The waves also traveled faster in the absence of the brain as assessed by the propagating duration of the wave between A7 and A1 (without brain, $1.7 \pm 0.25 \mathrm{~s}, n=6$; with brain, $12.7 \pm 0.90 \mathrm{~s}, n=8$; Fig. $1 C$ ). However, there was no detectable difference in the patterns of the waves between the two conditions, including the bilateral symmetry of the activity (Fig. 1D). A previous study also reported that forward calcium waves are more frequent in the isolated CNS without the brain (Pulver et al., 2015).

\section{Photoinhibition of MNs in A5 segment reduces the frequency} of the motor wave

Using the preparation described above, we next tried to manipulate $\mathrm{MN}$ activity locally and temporally using optogenetics while observing the motor waves by calcium imaging. To simultane- 
A

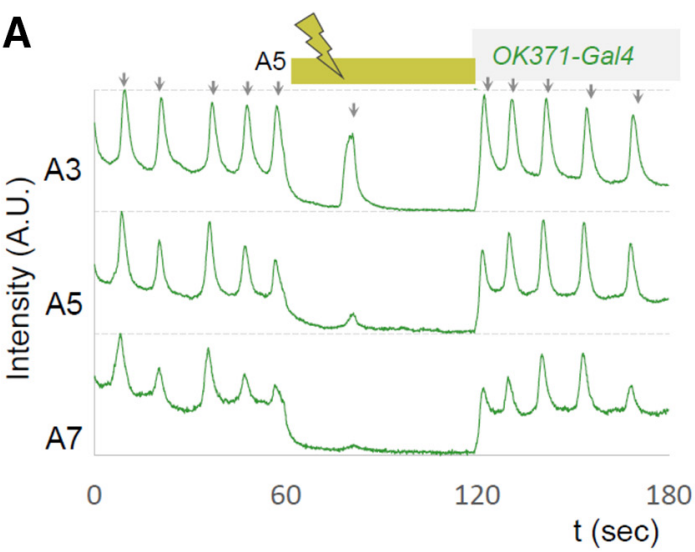

B

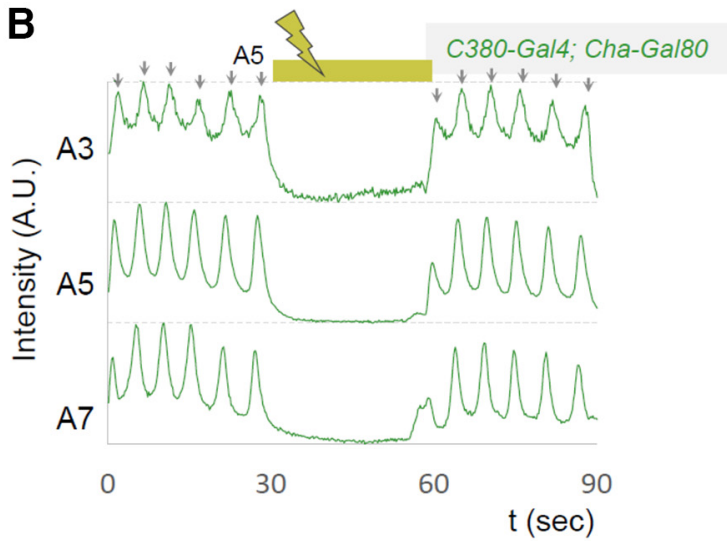

C

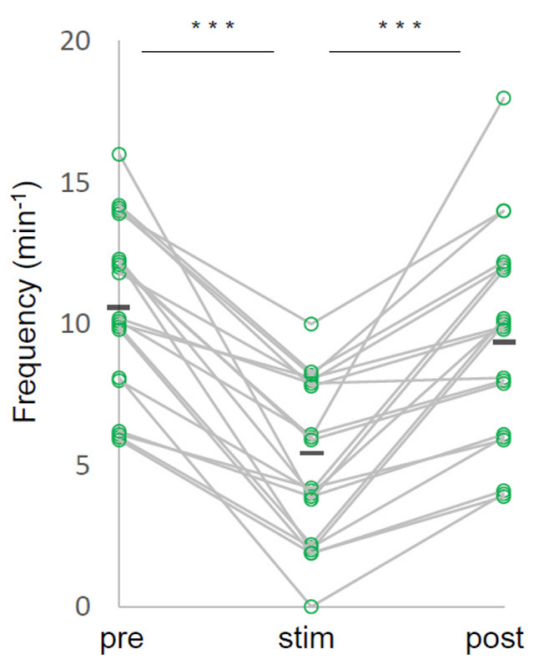

$\mathbf{E}$

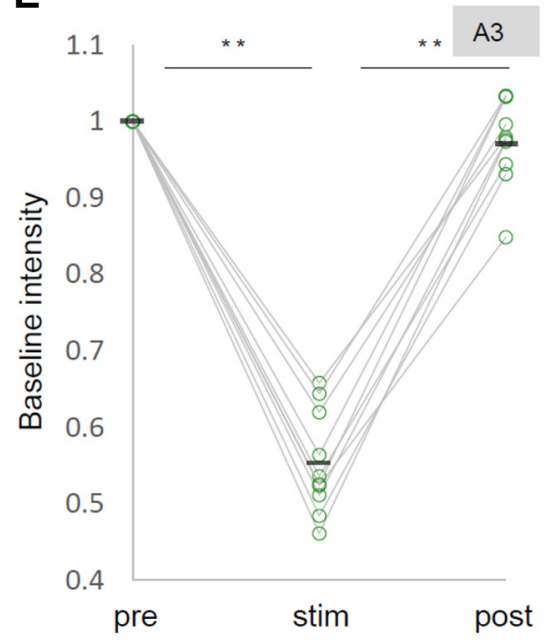

D 25

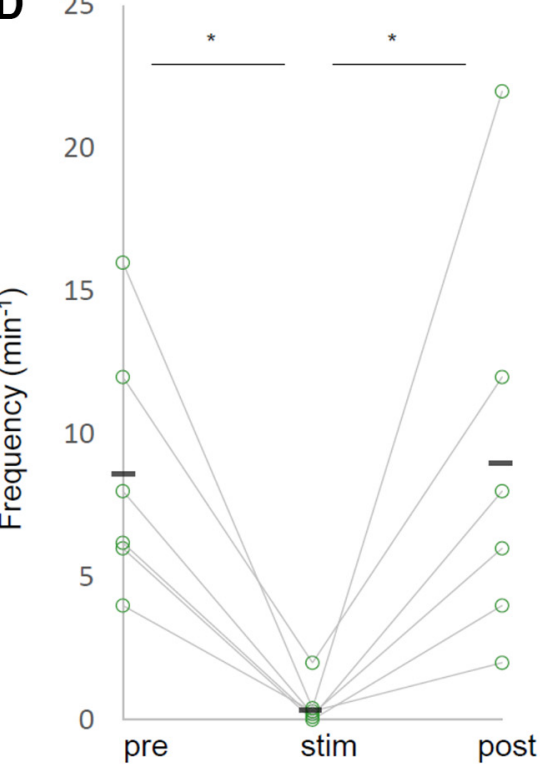

$\mathbf{F}$

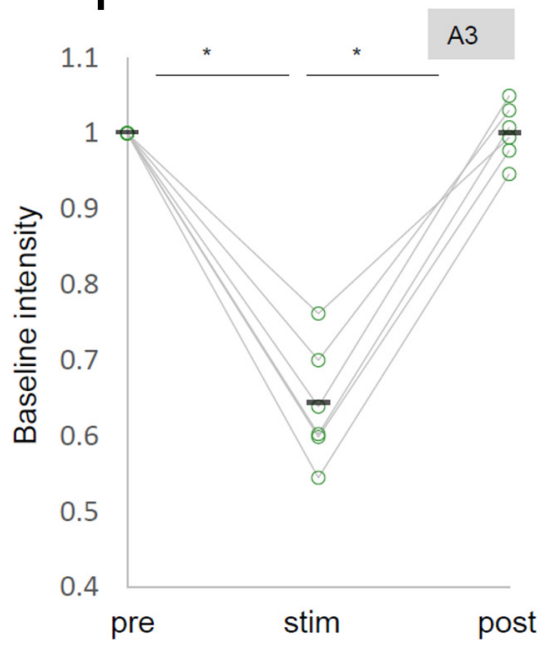

Figure 4. Decreased motor waves induced with independent MN Gal4 drivers. $\boldsymbol{A}-\boldsymbol{F}$, Representative plots of calcium imaging $(\boldsymbol{A}, \boldsymbol{B})$ and changes in the frequency of motor waves $(\boldsymbol{C}, \boldsymbol{D})$ and normalized baseline intensity in $A 3(\boldsymbol{E}, \boldsymbol{F})$ are shown as in Figure 3. OK371-Gal4 $(\boldsymbol{A}, \boldsymbol{C}, \boldsymbol{E})$ or $\left(380-G a l 4\right.$; Cha-Gal80 $(\boldsymbol{B}, \boldsymbol{D}, \boldsymbol{F})$ were used as drivers. ${ }^{*} p<0.05$; ${ }^{* *} p<0.01$; ${ }^{* * *} p<0.001$ (Wilcoxon signed-rank test).

ously photoinhibit and visualize MN activity, we expressed NpHR3::mCherry and GCaMP6m in MNs using a MN-specific Gal4 driver, OK6-Gal4 (Fig. 2F). With confocal microscopy, we applied spatially restricted yellow light for $60 \mathrm{~s}$ to a neuropile region containing one of the bilateral motor nerve roots in the A5 neuromere while calcium imaging $\mathrm{MN}$ activity by illuminating with blue light (Fig. 2A,B). Light stimulation was applied unilaterally to allow calcium imaging of MNs in the other side of the segment (Fig. $2 B, E$ ). Since all MNs project their axons to muscles via the nerve root, we expected that the optogenetic manipulation 


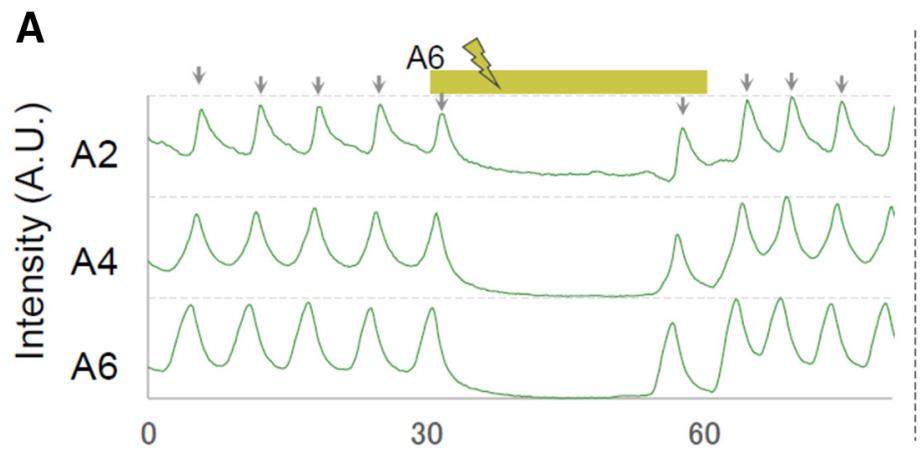

B

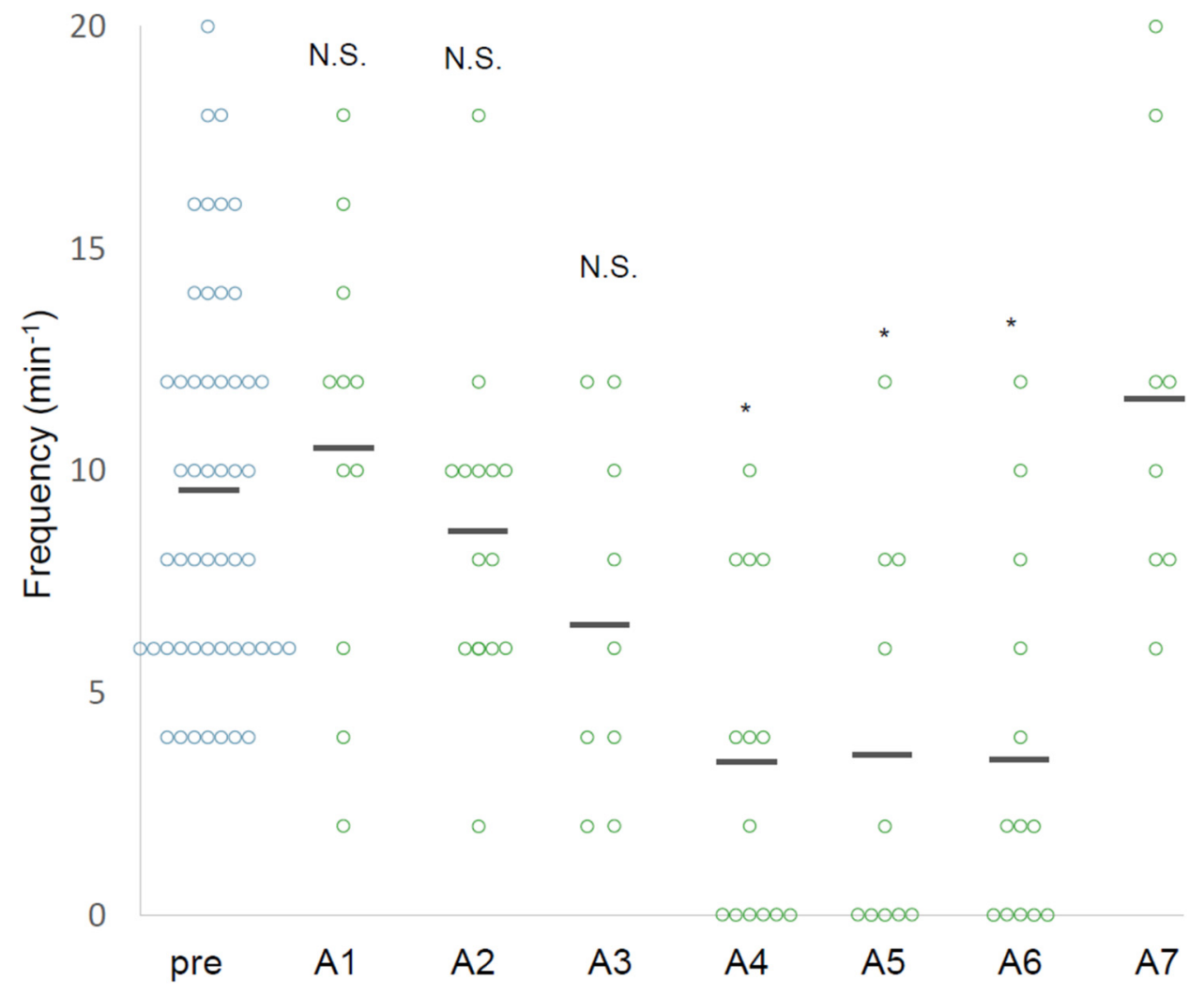

Figure 5. Segment specificity in the effects of local photoinhibition of MNs. $A$, Normalized fluorescent intensity in $A 2, A 4$, and A6 are plotted. Yellow bars indicate the duration of yellow light stimulation in A6 (30-60 s) and A2 (150-180 s). B, Frequency of motor waves before (pre) and during stimulation in each of the A1-A7 segments. ${ }^{*} p<0.05$ (Steel's test).

would affect a majority of MNs innervating the corresponding bodywall hemisegment. We used larvae with the same genotype but not fed with all-trans retinal (ATR) as a negative control group.

We found that the frequency of the motor waves was dramatically decreased during the local photoinhibition (number of waves per min before, during, and after the photostimulation, $\mathrm{ATR}^{+}, 10.4 \pm 1.65, n=10 ; 3.6 \pm 1.42, n=10 ; 10.0 \pm 1.69, n=$ 10 ; ATR $^{-}, 10.6 \pm 1.56, n=7 ; 10.3 \pm 1.48, n=7 ; 9.1 \pm 1.14, n=$ 7; Fig. $3 A, B$, Movies 1-3). Although the frequency was greatly reduced, the waves traveled along the segment normally when they occurred, albeit at a slightly higher speed (propagating duration from A6 to A4, prestimulation, $0.5 \pm 0.062 \mathrm{~s}$; during stimulation, $0.356 \pm 0.057 \mathrm{~s}$, $n=16$; Fig. $3 C$ ). Thus, the local optical perturbation interrupted generation but not propagation of motor waves.

We also found that upon light application, the GCaMP fluorescent intensity decreased in the contralateral side of the

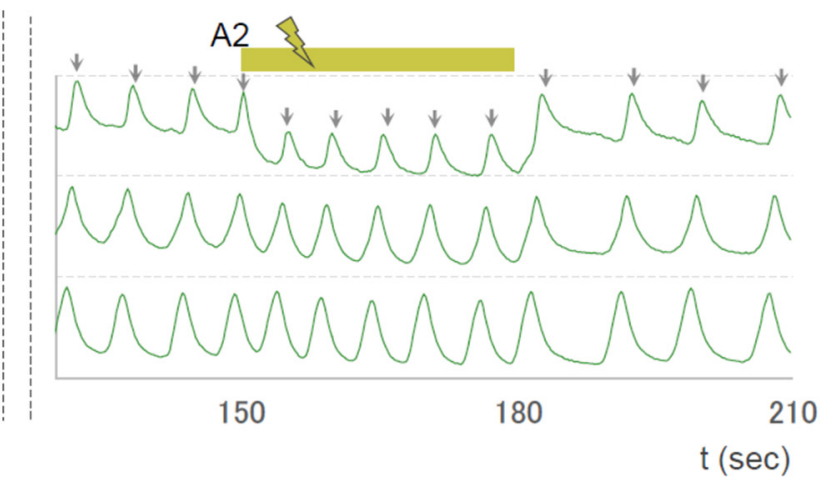

N.S.

Yellow bars indicate the d
genents. ${ }^{*} p<0.05$ (Steel's test)

manipulated segment A5 (Fig. 3 A, D,E, Movie 2). This may have occurred for a couple of reasons: (1) Some MNs extend their axon and/or dendrites contralaterally (Kim et al., 2009) and could be inhibited by the contralateral optical perturbation. (2) MNs in both sides of a segment could be electrically interconnected as has been shown for MNs in neighboring segments (Macleod et al., 2003). Furthermore, basal intensity of GCaMP fluorescence in other segments was also reduced upon the A5 photoinhibition (Fig. $3 F, G$ ). This suggests that inhibition of MN activity in A5 changes the basal activity level of MNs in other neuromeres. Together, these results show that local photoinhibition of MNs in A5 decreased the basal calcium level in MNs in the entire VNC and the frequency of motor waves. While expression driven by OK6-Gal4 is largely restricted to MNs in the ventral nerve cord, the Gal4 also drives expression in a small number of interneurons (Sanyal, 2009; our unpublished observation). We therefore tested 
A

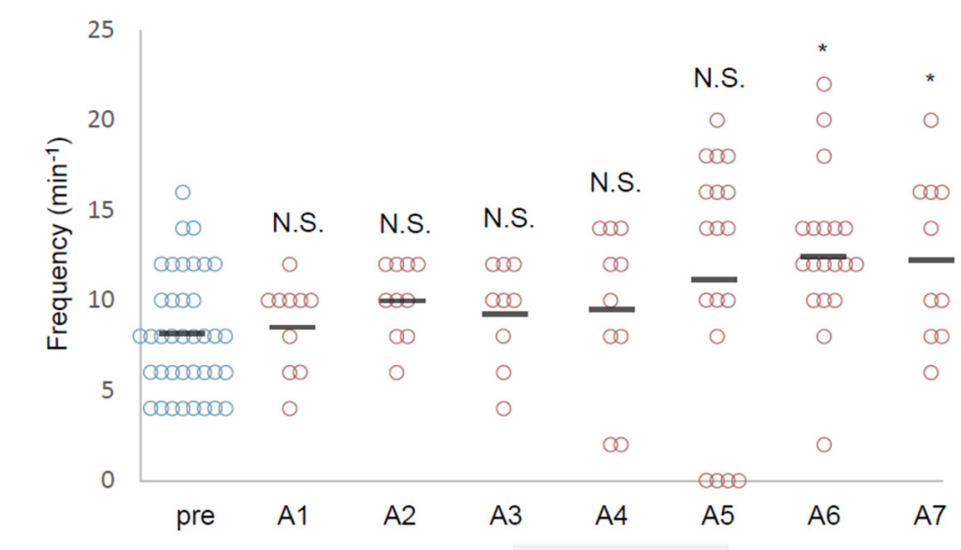

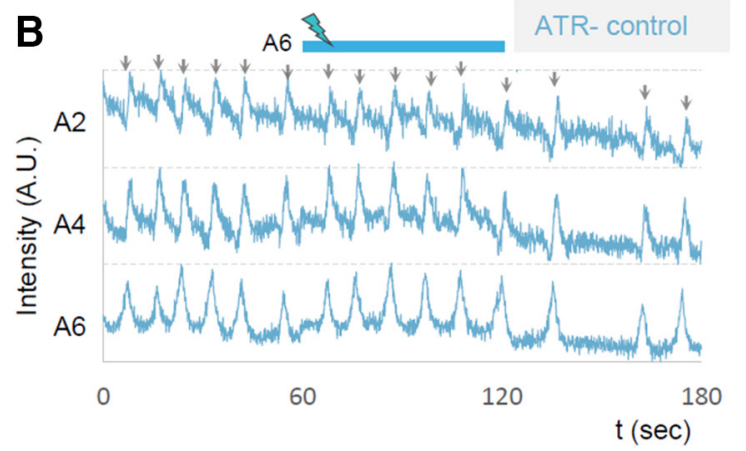

C

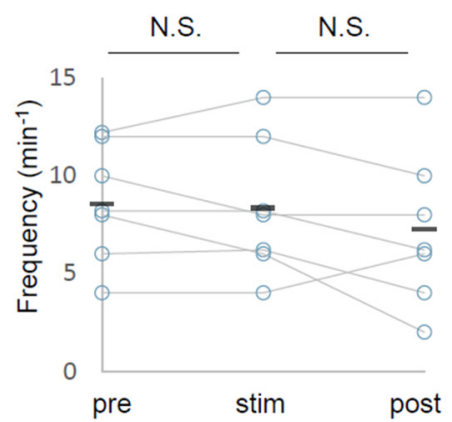

D

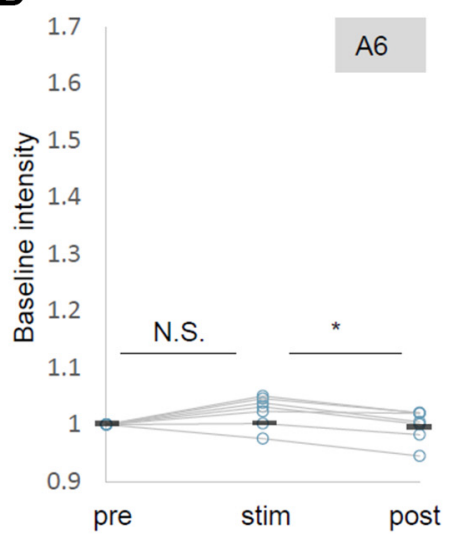

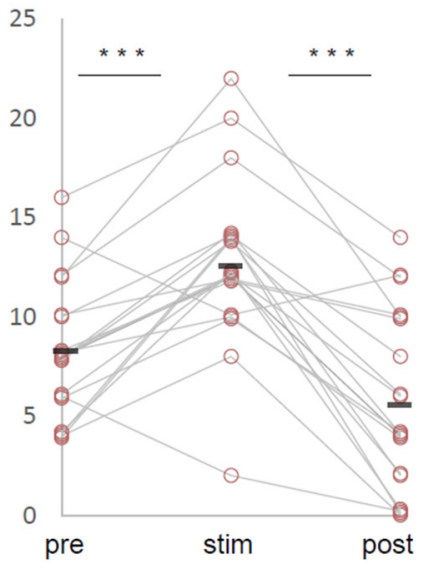

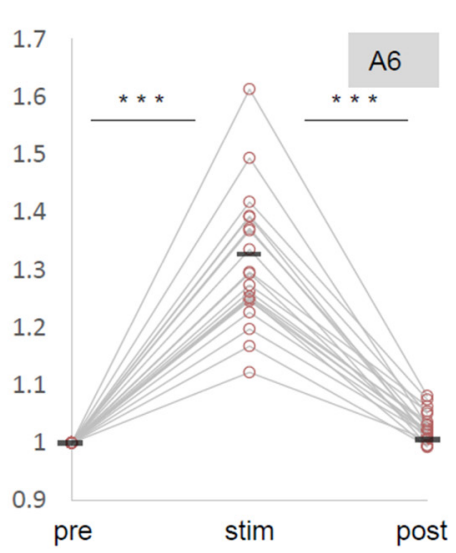

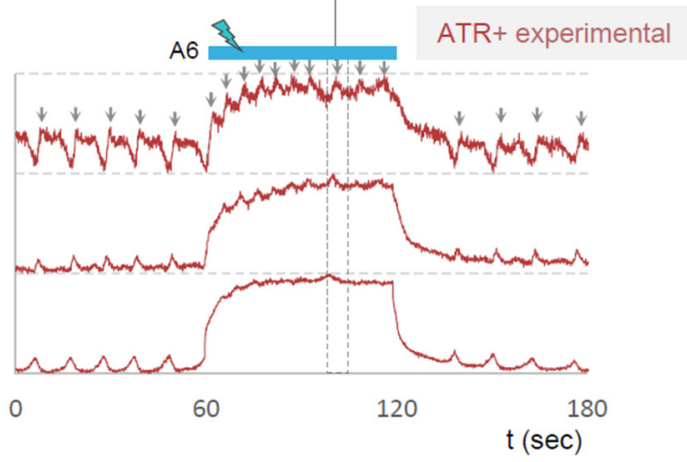

E
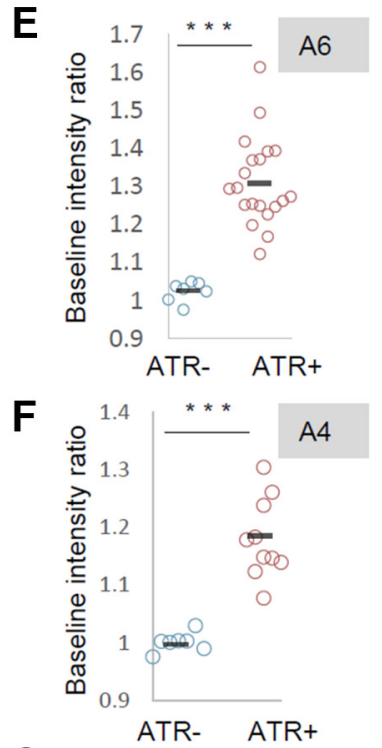

G

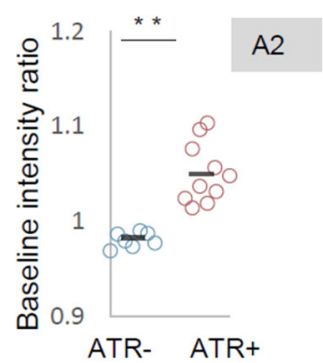

Figure 6. Photoactivation of MNs in posterior segments increased the frequency of motor waves. $\boldsymbol{A}$, Frequency of motor waves before (pre) and during photoactivation in segments A1 to A7. $\boldsymbol{B}$ - $\boldsymbol{G}$, Increased motor waves upon MN activation in A6. $\boldsymbol{B}$, Representative plots of normalized fluorescent intensity in control (ATR ${ }^{-}$, left) and experimental (ATR ${ }^{+}$, right) groups. ROls were made in a region surrounding the nerve root of $A 2, A 4$, and $A 6$ in the contralateral side of the optical manipulation as shown in Figure $2 D$. Blue bars (60-120 s) indicate the duration of light stimulation. $C-G$, Changes in the frequency of motor waves $(\boldsymbol{C})$ and normalized baseline intensity in $A 6(\boldsymbol{D}, \boldsymbol{E}), \mathrm{A4}(\boldsymbol{F})$, and A2 (G) upon optical activation of $A 6 .{ }^{*} p<0.05($ Steel's test; $\boldsymbol{A})$; ${ }^{*} p<0.05$, ${ }^{* * *} p<0.001$ (Wilcoxon signed-rank test; $\boldsymbol{C}, \boldsymbol{D}){ }^{* *} p<0.01,{ }^{* * *} p<0.001$ (Mann-Whitney $U$ test; $\boldsymbol{E}-\mathbf{G}$ ). 


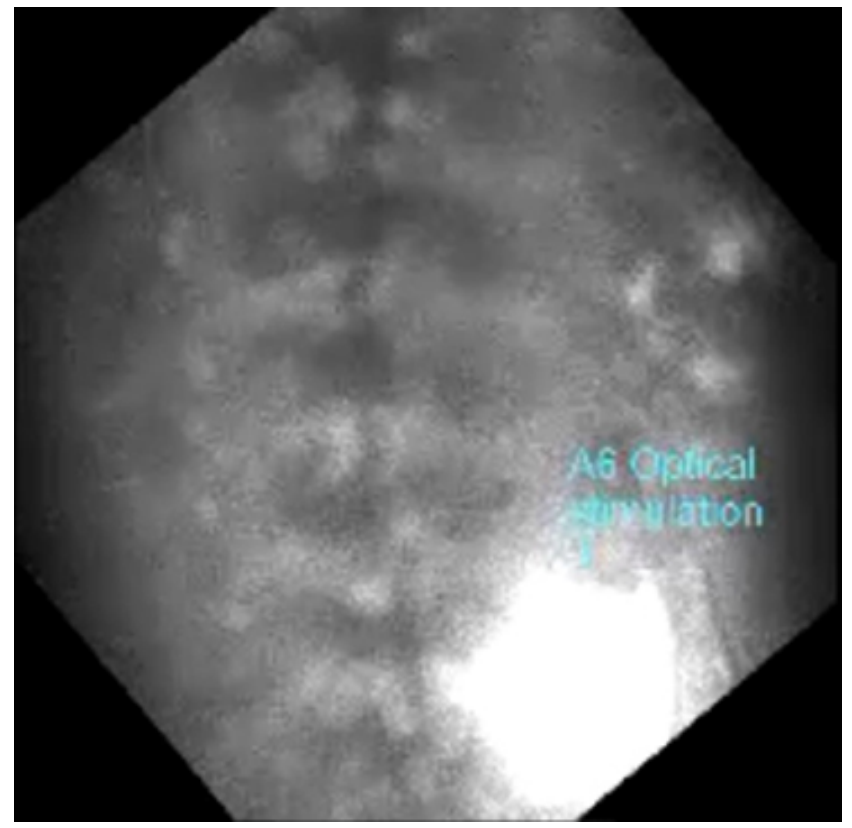

Movie 4. The effects of local photoactivation of A6 in an experimental $\left(\mathrm{ATR}^{+}\right)$larva. This movie is related to Figure 6 . The speed of the movie is $4 \times$ the speed of the raw data.

whether similar changes in $\mathrm{MN}$ activity were seen when other MN-Gal4 drivers, OK371-Gal4 and C380-Gal4; Cha-Gal80, were used, and found that it is the case (number of waves per minute before, during, and after the photostimulation, OK371-Gal4, $10.6 \pm 0.66,5.1 \pm 0.61,9.4 \pm 0.81, n=21$; C380-Gal4; ChaGal80, $8.7 \pm 1.84,0.3 \pm 0.33,9.0 \pm 2.96, n=6$; Fig. 4$)$. These results strongly support the notion that MNs, not interneurons, were responsible for the phenotype.

\section{Segment specificity in the effects of local photoinhibition}

We next examined whether photoinhibition of MNs in segments other than A5 would have the same effects. We applied unilateral photoinhibition to each neuromere from A7 to A1 and found that the manipulation in a middle segment (A4, A5, or A6), but not in a more anterior (A1, A2, or A3) or posterior (A7) segment, reduced the frequency of motor waves (Fig. 5). Thus, local photoinhibition of MNs decreases the wave frequency in a segmentspecific manner. The observed segmental difference was not due to differences in the expression of $\mathrm{NpHR} 3$, since it was uniformly expressed across segments (data not shown).

\section{Local photoactivation of MNs in specific segments increases the frequency of motor waves}

Next we examined the effects of local photoactivation of MNs. To simultaneously photoactivate and visualize MN activity, we expressed ChR2 (T159C) and RCaMP in MNs and applied blue light unilaterally and locally for optogenetic activation while illuminating with yellow light for calcium imaging (Fig. 2C, D, G). As we observed segmental specificity in the effects of the photoinhibition, we studied the effects of local photoactivation applied to each of the A1-A7 segments. We found that activation of MNs in a posterior segment (A6 or A7), but not in other segments (A1A5), increased the frequency of the motor waves (Fig. 6A, Movie 4). Figure $6 B-G$ shows the results of A6 photoactivation. Upon photoactivation, wave frequency was specifically increased in the experimental but not in the control group (number of waves per minute before, during, and after the photostimulation, $\mathrm{ATR}^{+}$, $8.2 \pm 0.79 ; 12.7 \pm 0.99 ; 5.7 \pm 1.05$ events, $n=19 ; \mathrm{ATR}^{-}, 8.6 \pm$ $1.13 ; 8.3 \pm 1.34 ; 7.1 \pm 1.34, n=7$; Fig. $6 B, C)$. As we observed for local $\mathrm{MN}$ inhibition, the local photoactivation also changed the basal $\mathrm{Ca}^{2+}$ level of MNs in the entire CNS. As shown in Figure $6 D-G$, upon local photoactivation in A6, RCaMP fluorescent intensity in MNs in the contralateral side of A6 and other segments increased. Similar results were obtained for A7 photostimulation (data not shown). Thus, local photoactivation of MNs in A6 and A7 increased the basal $\mathrm{Ca}^{2+}$ level of MNs in the entire CNS and the frequency of motor waves.

We also used RRA-Gal4-F (Fujioka et al., 2003), which drives expression in aCC and RP2 MNs, and CQ2-Gal4 (Landgraf et al., 2003), which drives expression in U MNs, to ask whether photostimulation of small subsets of MNs show the same effects as the manipulation of all MNs. We found that photoactivation of $U$ MNs with CQ2-Gal4 (but not that of aCC and RP2 MNs with $R R A-G a l 4-F)$ in A6 increased the frequency of the motor waves as seen when OK6-Gal4 driver was used (Fig. 7; number of waves per minute before, during, after photostimulation, $R R A, 10.9 \pm$ $1.06,11.4 \pm 1.36,9.71 \pm 1.34, n=7$; CQ2, $13.0 \pm 2.30,16.3 \pm$ $2.09,12.25 \pm 2.02, n=8$ ). The results provide further evidence that manipulation of MNs, not interneurons, caused the change in motor pattern, and partly identified specific MNs (Us) involved in the process. Photoinhibition with the RRA-Gal4-F or CQ2-Gal4 driver had no effects on the frequency of the motor waves (data not shown).

\section{Gap junctions mediate the changes in motor wave frequency} Local photoinhibition and photoactivation data together indicate that local changes in MN activity can change the activity level of the entire motor circuit. This suggests that information regarding MN activity is transmitted to the central circuits and causes changes in their dynamics. Gap junctions are good candidates for mediating this process, since they allow global neuronal signaling among a group of neurons. In Drosophila larvae, it has been suggested that MNs are electrically coupled via gap junctions between neighboring segments (Macleod et al., 2003). In contrast, chemical transmission among MNs or from MNs to interneurons has not been reported (Landgraf et al., 2003). We used shak $B^{2}$ mutants, which lack two splicing variants $[\operatorname{ShakB}(\mathrm{N})$ and ShakB(N+16)] of ShakB (innexin8), a major component of gap junctions in Drosophila larval CNS (Stebbings et al., 2002; Phelan et al., 2008), and tested whether the effects of the photomanipulation were abolished in the mutants. We also studied an allele of Ogre (innexin1), another innexin known to be expressed in the late embryonic CNS (Stebbings et al., 2002). We first applied local inhibition in the gap-junction mutants. The frequency of motor waves in the absence of photostimulation was normal in both mutants (number of waves per minute, shakB ${ }^{2}, 7.1 \pm 1.11$; control, $7.5 \pm 0.98 ; p=0.80 ;$ ogre $^{2}, 11.4 \pm 1.13$; control, $9.14 \pm 1.37$; $p=0.24$ ). However, the photoinhibition in A5, which normally reduces the frequency of motor waves, did not change the frequency in shakB ${ }^{2}$ or ogre ${ }^{2}$ mutants compared to control (number of waves per minute before and during the photostimulation, shakB $B^{2}, 7.8 \pm 1.22,8.2 \pm 1.35, n=9$; control, $10.0 \pm 1.66,3.6 \pm$ $1.4, n=10 ;$ ogre $^{2}, 11.25 \pm 0.996,8 \pm 0.756, n=8$; control, $9.14 \pm$ $1.37,3.43 \pm 1.42, n=7$; Fig. $8 A, B)$. Thus, both ShakB and Ogre are required for the photoinhibition to change the wave frequency. Interestingly, the photoinhibition effectively reduced the basal $\mathrm{Ca}^{2+}$ level of MNs in the manipulated A5 and in other 

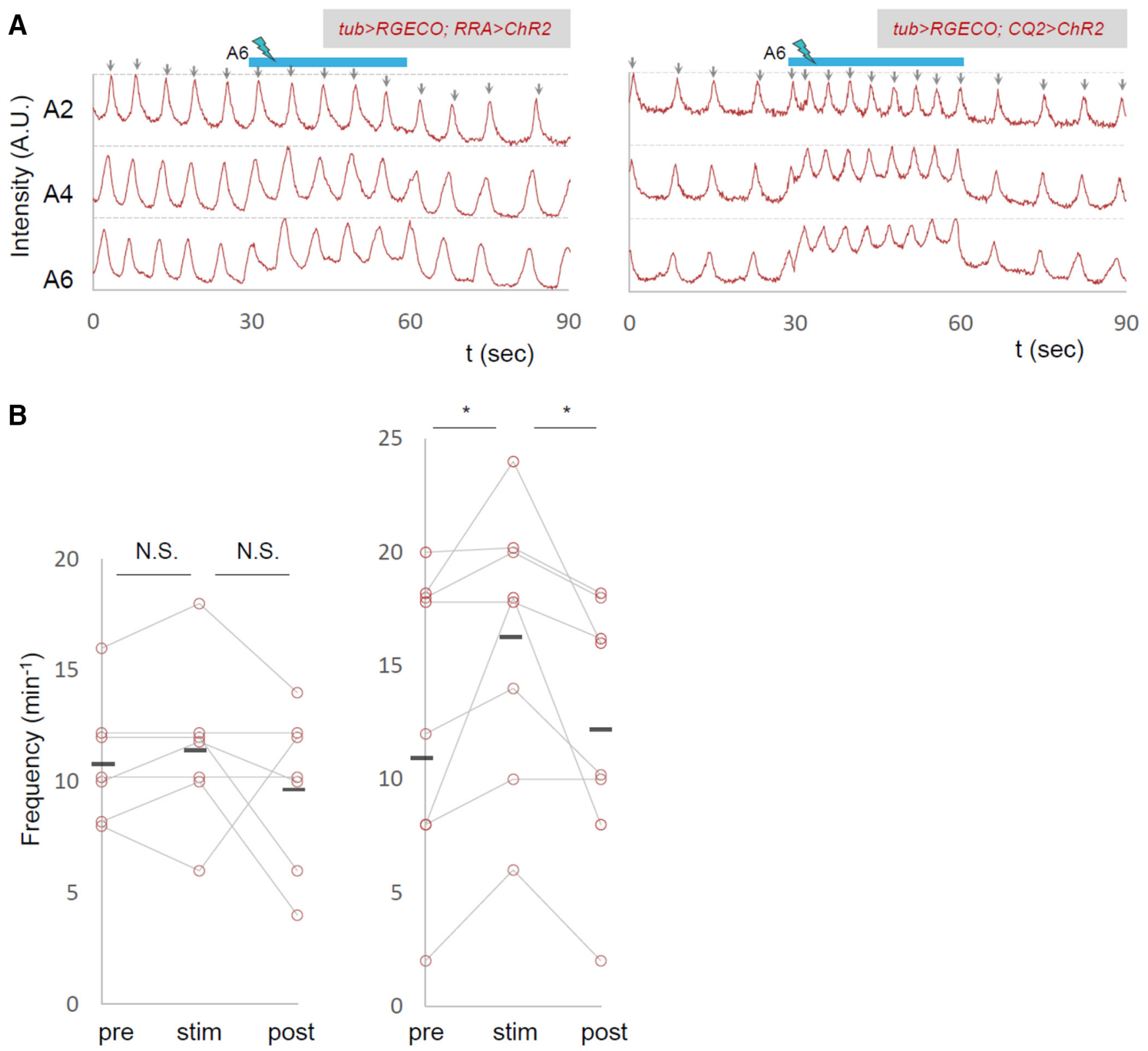

Figure 7. Photoactivation of U MNs increased the frequency of motor waves. The effects of local photoactivation of small subsets of MNs. A, Representative plots of normalized fluorescent intensity upon photostimulation of ChR2, whose expression is driven by RRA-Gal4 (left) and CQ2-Gal4 (right). ROls were made in a region surrounding the nerve root of A2, A4, and A6 in the contralateral side of the optical manipulation as shown in Figure 2D. Blue bars $(30-60 \mathrm{~s})$ indicate the duration of light stimulation. $\boldsymbol{B}$, Changes in the frequency of motor waves upon optical manipulation are plotted. ${ }^{*} p<0.05$ (Wilcoxon signed-rank test).

segments in these mutants as in the wild-type (Fig. $8 C, D$ ). This suggests that information regarding the changes in $\mathrm{MN}$ activity in A5 can be transmitted to MNs in other segment in the mutants, possibly via gap junctions not including ShakB or Ogre, or through other mechanisms. Thus, shak $B^{2}$ and ogre ${ }^{2}$ mutations compromise specific aspects of MN signaling that regulate the frequency of motor waves.

Does ShakB or Ogre function in MNs to mediate the MN signaling? To address this, we tested the effects of $\mathrm{MN}$-specific knockdown of shakB or ogre by using RNAi. We used the shakB$R N A i$ and ogre-RNAi constructs that produce short hairpin RNAs and have no or few potential off-targets (Ni et al., 2011). A previous study showed that RNAi knockdown of ShakB or Ogre using these constructs changed the amplitude of the compound action potential recorded in response to sound from the base of the antenna (sound-evoked potential; Pézier et al., 2016), suggesting that these RNAi constructs are effective. We found that $\mathrm{MN}$-specific knockdown of shakB compromised the effects of the photoinhibition as observed in the mutants (number of waves per minute before and during the photostimulation, shakB, $12.5 \pm 1.5,10.25 \pm 1.5, n=8$; control, $10 \pm 1.7$, $3.6 \pm 1.4$; Fig. $8 E)$. This suggests that ShakB functions in MNs to regulate the wave frequency. In contrast, $\mathrm{MN}$-specific knock-down of ogre had no effect on the photoinhibition, suggesting that Ogre functions in other cells than MNs, possibly interneurons (10.4 \pm 2.48 , $3.2 \pm 1.36, n=5$; Fig. $8 E$ ).

We also examined the effects of photoactivation in $\operatorname{shakB} B^{2} \mathrm{mu}-$ tants. As was the case for photoinhibition, photoactivation in A6 that normally increases the frequency of motor waves did not do so in the mutants (number of waves per minute before and during the pho- 


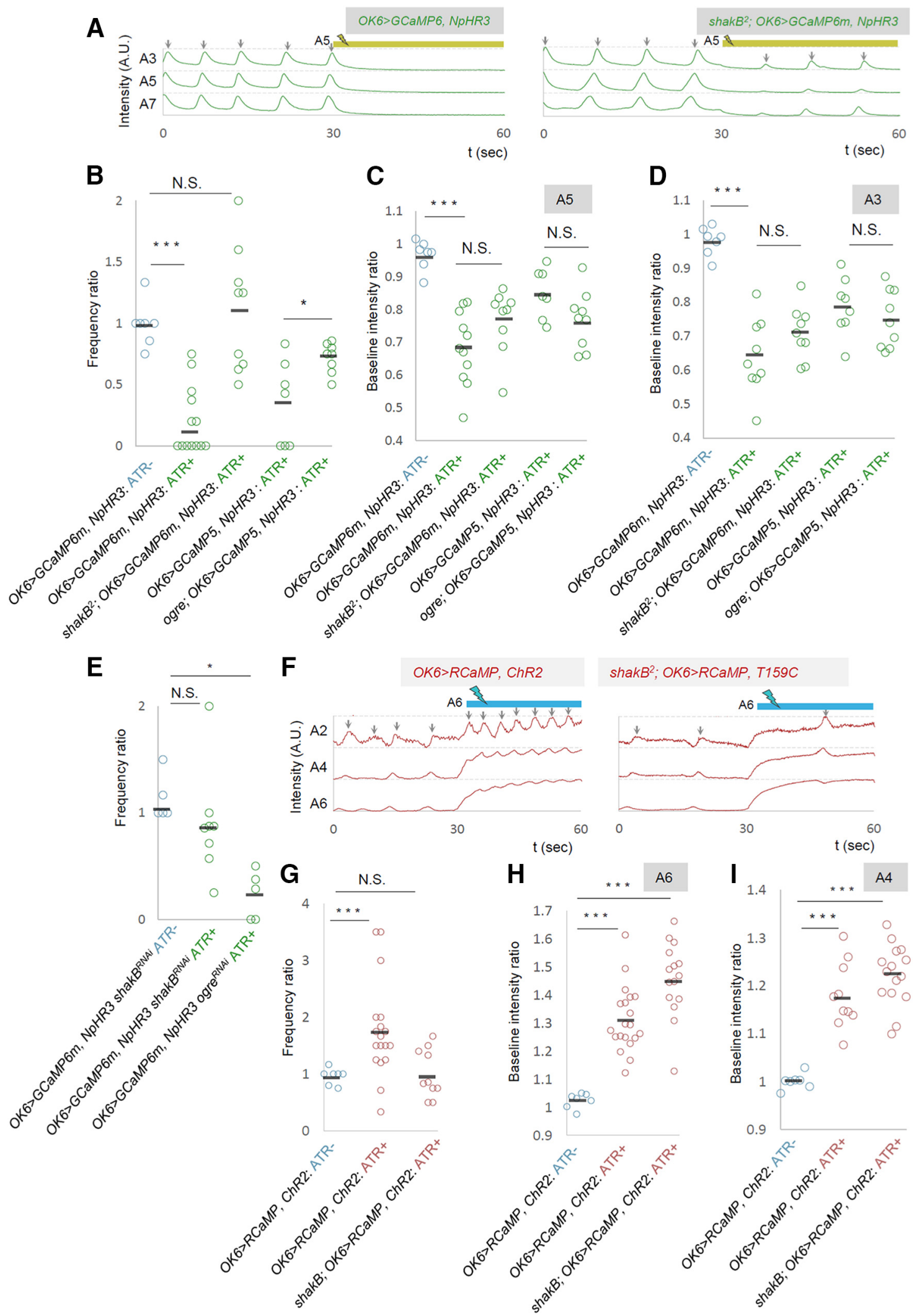

Figure 8. shakB and ogre are involved in the changes in wave frequency. $A-D$, The effects of local photoinhibition in shak $B^{2}$ or ogre ${ }^{2}$ mutants. $A$, Representative plots of $G C a M P$ fluorescent intensity in control (left) and shak $B^{2}$ (right) are shown as in Figure $2 A$. $\boldsymbol{B}-\boldsymbol{D}$, Frequency ratio of motor waves $(\boldsymbol{B})$ and baseline fluorescent intensity in $A 5(\boldsymbol{C})$ and $A 3(\boldsymbol{D})$. $\boldsymbol{E}$, The frequency ratio of local photoinhibition in shakB and ogre RNAi knockdown in MNs (driven by OK6-Gal4). $\boldsymbol{F}-\boldsymbol{I}$, The effects of local photoactivation in shak ${ }^{2}$ mutants. $\boldsymbol{F}$, Representative plots of RCaMP fluorescent intensity in control (left) and shakB ${ }^{2}$ (right). $\mathbf{G}-\boldsymbol{I}$, Frequency of motor waves $(\boldsymbol{G})$ and baseline fluorescent intensity in $A 6(\boldsymbol{H})$ and $A 4(\boldsymbol{I}) .{ }^{*} p<0.05$; ${ }^{* * *} p<0.001$ (Mann-Whitney $U$ test). 

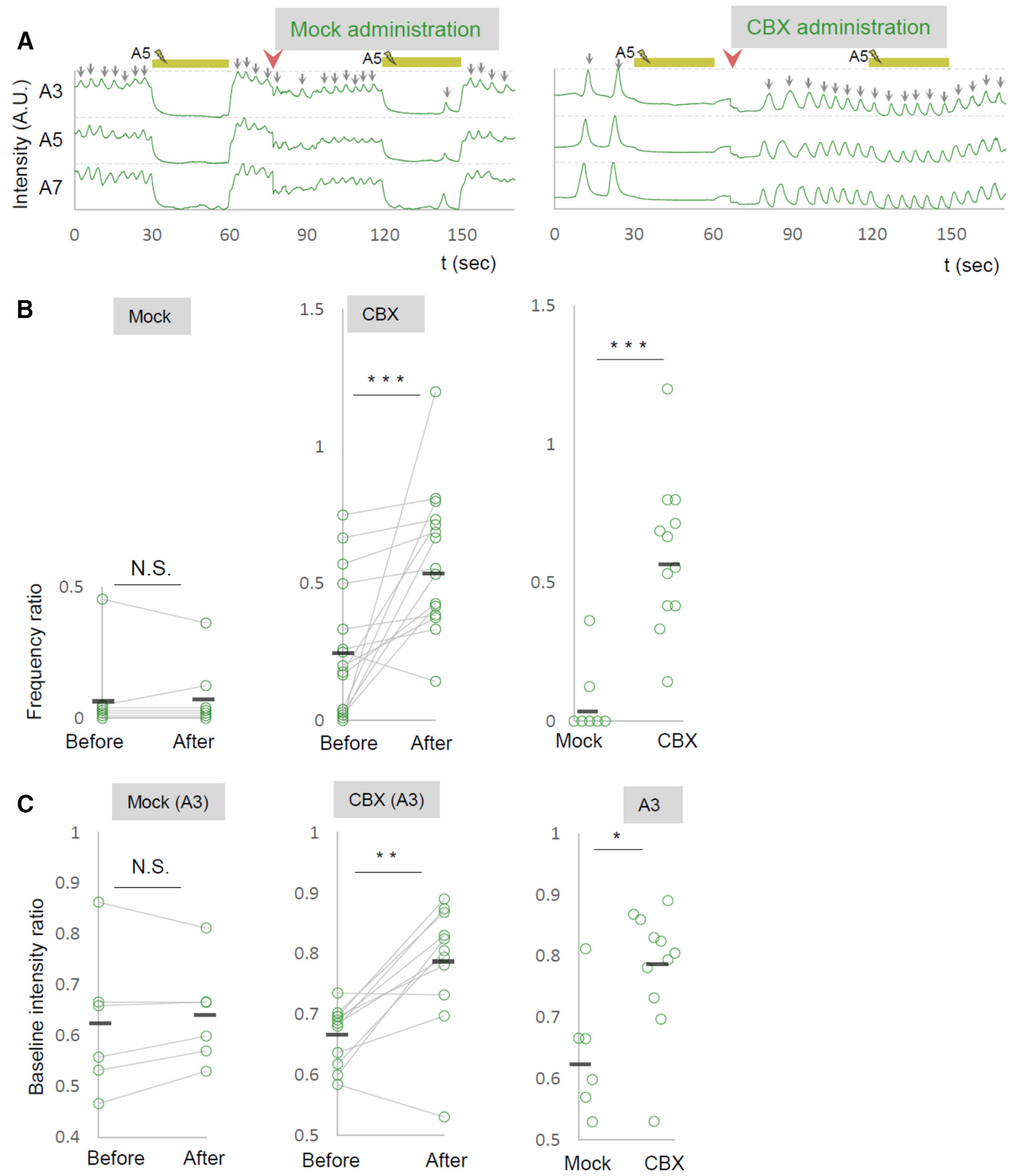

Figure 9. A gap-junction blocker acutely reverts the effects of photoinhibition. $A-C$, The effects of CBX administration on the local photoinhibition of MNs. $A$, Representative plots of GCaMP fluorescent intensity in control (left) and upon (BX administration (right) are shown. $\boldsymbol{B}, \boldsymbol{C}$, Changes in the frequency of motor waves $(\boldsymbol{B})$ and of baseline fluorescent intensity in $A 3$ contra neuropile (C), upon photoinhibition of MNs in $A 5$, are shown for before and after (BX administration. ${ }^{* *} p<0.01$, ${ }^{* *} p<0.001$ (Wilcoxon signed-rank test; $B, C$, left, middle); ${ }^{*} p<0.05,{ }^{* * *} p<0.001$ (Mann-Whitney $U$ test; $\boldsymbol{B}, \boldsymbol{C}$, right).

tostimulation: $\operatorname{shakB} B^{2}, 7.6 \pm 1.29 ; 7.4 \pm 1.19, n=10$; control, $8.21 \pm$ $0.79 ; 12.7 \pm 0.99, n=19$; Fig. $8 F, G)$. In contrast, global change in the basal $\mathrm{Ca}^{2+}$ level of MNs occurred in the mutants, again as observed for photoinhibition (Fig. $8 H, I$ ).

\section{A pharmacological blocker of gap junctions acutely inhibits} the MN signaling

Gap junctions are known to play critical roles not only in shaping the information flow in mature neural circuits, but also during 
development of the nervous system (Juszczak and Swiergiel, 2009). To test whether the defects seen in shakB or ogre mutants described above reflect the role(s) of these innexins in development or neural signaling in matured circuits, we used a pharmacological blocker, CBX, to acutely inhibit the function of gap junctions. We found that administration of CBX reverted the effects of the photoinhibition of MNs on motor wave generation, as was observed in shakB or ogre mutants (ratio of wave number during/before photostimulation; before CBX administration, $0.2570 \pm 0.064$; after CBX administration, $0.5833 \pm 0.065$; Fig. 9A,B). Thus, gap junctional communication is required in the mature circuits to mediate the $\mathrm{MN}$ signaling. Interestingly, unlike shakB or ogre mutation, administration of CBX also suppressed the decrease in the basal $\mathrm{Ca}^{2+}$ level in MNs in a distant neruomere (A3) induced by the local $\mathrm{MN}$ photoinhibition in A5 (Fig. 9C). This suggests that gap junctions containing innexin(s) other than ShakB and Ogre mediate this aspect of $\mathrm{MN}$ signaling. If the intersegmental $\mathrm{MN}$ signaling is mediated by direct $\mathrm{MN}-\mathrm{MN}$ gap junctions, MNs in one segment should have physical contacts with MNs in neighboring segments. Indeed, we found such membrane contacts in ssTEM reported previously (Ohyama et al., 2015; Zwart et al., 2016; Fig. 10). Although the resolution of the EM reconstruction is not high enough to anatomically detect gap junctions in the contacts, the observation is consistent with the idea that MNs communicate with each other via gap junctions.

\section{Discussion}

In our previous study (Inada et al., 2011), we locally and transiently inhibited $\mathrm{MN}$ activity in one or a few segments during peristalsis of dissected larvae and showed that activity manipulation halts peristalsis. This indicates that $\mathrm{MN}$ activity is required for the motor activity wave to propagate along the VNC and suggests the presence of retrograde signaling from $\mathrm{MNs}$ to the central circuits. However, since dissected larvae were used, the possibility that the signaling was instead mediated via the sensory feedback of muscular contraction could not be excluded. Furthermore, the mechanism of the retrograde signaling remained unknown.

In this study, we built a new experimental system that allowed us to study the direct causal relationship between the manipulation of MN activity and changes in neural dynamics in the motor circuits with superior spatial and temporal resolution. We applied optical perturbations for a longer period and in a more systematic manner than in the previous study and analyzed their effects on the global circuit activity. Using the new experimental system, we extended the previous study by showing that (1) MN outputs within the CNS, not mediated by sensory feedback, are
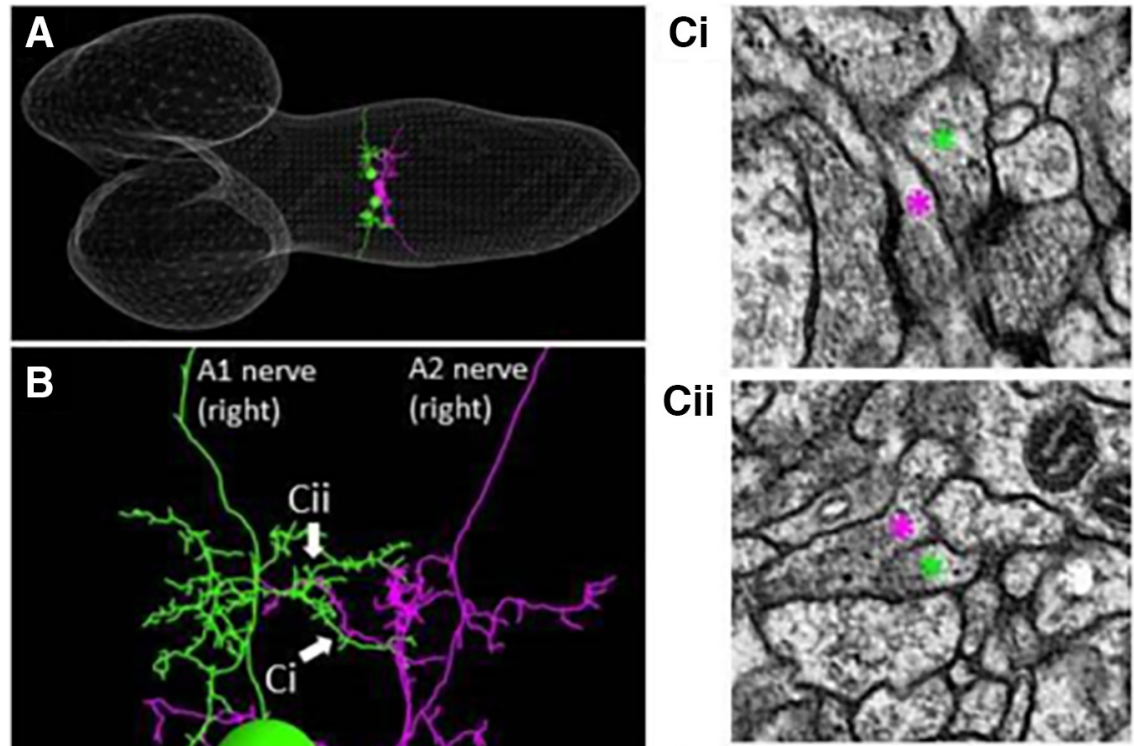

Figure 10. Intersegmental contacts between MNs. A, A reconstructed image of ssTEM of the CNS of a first instar (white line meshes). A pair of RP2 motor neurons in the A1 segment (green) and RP3 motor neurons in the neighboring A2 segment (purple) $\boldsymbol{C}$-Civ, Electromicroscopic images of the intersegmental contacts indicated by arrows in $\boldsymbol{B}$. Neurites of the MNs are depicted by corresponding colored asterisks (purple and green). Scale bar, $100 \mathrm{~nm}$.

critical for motor wave regulation, (2) there is segmental difference in the role of the MN outputs, and (3) the MN signaling is mediated by gap junctions. Below, we discuss each of these findings.

\section{Contribution of local motoneuronal activity to generation of motor waves}

Here, we showed that manipulation of motor neuronal activity in just one segment robustly affects the output of the entire motor network in Drosophila larvae. Optical inhibition or activation of MNs in a single segment decreased or increased, respectively, the calcium level of MNs in distant neuromeres. Furthermore, these perturbations strongly affected the frequency of motor waves. Thus, changes in MN activity in one segment affect the activity level and wave generation of the entire motor system.

It should be noted that in the isolated VNC preparation used in this study, peripheral nerves with motor activity output and 
sensory feedback input were severed. Local changes in MN activity therefore influenced the activity of distant MNs through intersegmental neural connections within the CNS, not via sensory feedback. Thus, our results establish the presence of retrograde signaling from MNs that is critical for motor pattern regulation. The identity of the synaptic connections mediating the signaling is currently unknown. They could be direct MN-MN connections, or they may also involve coupling between MNs and interneurons (Kohsaka et al., 2014; Heckscher et al., 2015; Itakura et al., 2015; Fushiki et al., 2016).

\section{Role of gap junction-mediated retrograde $\mathrm{MN}$ signaling in motor control}

Electrical synapses are commonly found in the nervous systems of vertebrates and invertebrates (Marder, 1998; Pereda, 2014). In particular, electrical coupling mediated by gap junctions has been implicated in motor pattern control in various systems (Marder and Calabrese, 1996; Tresch and Kiehn, 2000; Li et al., 2009). In this study, we showed that gap junctions are involved in the retrograde MN signaling controlling motor wave frequency in Drosophila larvae. Local photomanipulation of MNs that would normally increase or decrease wave frequency had no effect in shakB ${ }^{2}$ and ogre $^{2}$ mutants. This suggests that electrical synapses including ShakB and Ogre mediate the $\mathrm{MN}$ signaling controlling motor frequency. In contrast, CBX administration but not shakB ${ }^{2}$ or ogre ${ }^{2}$ mutation abolished the calcium level changes of distant MNs induced by the activity manipulation, suggesting that innexins other than those deleted in shak $B^{2}$ or ogre ${ }^{2}$ mediate this aspect of motoneuronal communication (eight innexin genes are present in the Drosophila genome; Bauer et al., 2005; Phelan et al., 2008). It should also be noted that wave generation normally occurred in the isolated VNCs of shakB ${ }^{2}$ and ogre ${ }^{2}$ mutants. There was also no obvious abnormality in the locomotion of the shakB $B^{2}$ or ogre ${ }^{2}$ larvae (data not shown). These observations suggest that ShakB and Ogre-mediated MN signaling is part of redundant pathway(s) regulating motor waves. Only upon optical perturbation are the role of $\mathrm{MN}$ signaling in wave generation and involvement of ShakB and Ogre manifested.

Previously, Song et al. (2016) reported the existence of electrical coupling between MNs and the premotor excitatory V2a interneurons, a neuronal class that provides a major drive for $\mathrm{MNs}$ during locomotion in zebrafish (Song et al., 2016). Hyperpolarizing or depolarizing MNs decreased or increased the firing activity of V2a interneurons. Furthermore, selective inhibition of MNs during locomotion interrupted the recruitment of V2a interneurons and decreased the frequency of locomotion. Thus, control of locomotor circuits by gap junction-mediated retrograde MN signaling may be an evolutionarily conserved mechanism used in both invertebrates and vertebrates.

\section{Segment specificity in the MN signaling}

An interesting feature of MN signaling revealed in this study is segment specificity. On the one hand, inhibition of MNs in A4, A5, or A6, but not other segments, reduced the motor wave frequency. On the other hand, activation of MNs in A6 or A7, but not other segments, increased the frequency of the wave. This segmental discord with regard to the MN signaling may contribute to the regulation of the wave initiation. How can gap junctions selectively mediate one type of activity change but not another? For example, how can the decline but not the elevation in activity level of MNs in the A4 or A5 segment affect the wave frequency? One possibility is the involvement of rectifying electrical synapses. Rectifying electrical synapses have been found in both vertebrates and invertebrates and can mediate unidirectional synaptic transmission in a voltage-dependent manner. Rectifying electrical synapses are often composed of a heteromeric assembly of gap junction proteins on each side of the apposing neurons (Marder, 1998; Rela and Szczupak 2004; Pereda, 2014). A role of ShakB in rectification has been shown in the giant fiber system of adult Drosophila. Two splicing forms of ShakB, $\operatorname{ShakB}(\mathrm{N})$ and $\operatorname{ShakB}(\mathrm{N}+16)$, are expressed in the presynaptic and postsynaptic sites of the giant synapse, respectively. When expressed in neighboring oocytes, these two ShakB variants form heterotypic channels that are asymmetrically gated by voltage. Since our RNAi knockdown experiments showed that shakB, but not ogre, is required in MNs to mediate the retrograde signaling, an interesting possibility is the involvement of heterotypic channels composed of ShakB in MNs and Ogre in interneurons. Future studies are necessary first to identify the target neurons that receive the retrograde $\mathrm{MN}$ signaling and then to study whether the relevant electrical synapses are indeed rectified. Revealing the information flow mediating the $\mathrm{MN}$ retrograde signaling will provide valuable insights on how intersegmentally coordinated motor patterns are generated in this and other systems. The experimental system established in this study can also be applied more generally to study the input-output relationship among the component neurons in this system. While the GAL4/UAS system alone was sufficient to express both GCaMP/RGECO and NpHR/ChR2 in MNs in this study, the introduction of another expression system such as the LexA system (Lai and Lee, 2006) will allow expression of GCaMP/RGECO and NpHR/ChR2 in different classes of neurons, including interneurons. This will allow us to study the influence of the optogenetic manipulation of one class of neurons on the activity of others. The functional analyses may also be combined with the circuit diagram elucidated by ongoing EM reconstruction (Heckscher et al., 2015; Ohyama et al., 2015; Fushiki et al., 2016; Schneider-Mizell et al., 2016) and theoretical models (Gjorgjieva et al., 2013; Davies et al., 2015; Hernandez-Nunez et al., 2015). We anticipate that such systematic analyses will elucidate fundamental mechanisms of how central circuits coordinate intersegmental movements.

\section{References}

Aberle H, Haghighi AP, Fetter RD, McCabe BD, Magalhães TR, Goodman CS (2002) Wishful thinking encodes a BMP type II receptor that regulates synaptic growth in Drosophila. Neuron 33:545-558. CrossRef Medline

Akerboom J, Carreras Calderón N, Tian L, Wabnig S, Prigge M, Tolö J, Gordus A, Orger MB, Severi KE, Macklin JJ, Patel R, Pulver SR, Wardill TJ, Fischer E, Schüler C, Chen TW, Sarkisyan KS, Marvin JS, Bargmann CI, Kim DS, et al. (2013) Genetically encoded calcium indicators for multi-color neural activity imaging and combination with optogenetics. Front Mol Neurosci 6:2. Medline

Bauer R, Löer B, Ostrowski K, Martini J, Weimbs A, Lechner H, Hoch M (2005) Intercellular communication: the Drosophila innexin multiprotein family of gap junction proteins. Chem Biol 12:515-526. CrossRef Medline

Blankenship AG, Feller MB (2010 Jan) Mechanisms underlying spontaneous patterned activity in developing neural circuits. Nat Rev Neurosci 11:18-29. CrossRef Medline

Brand AH, Perrimon N (1993) Targeted gene expression as a means of altering cell fates and generating dominant phenotypes. Development 118: 401-415. Medline

Brodfuehrer PD, Debski EA, O’Gara BA, Friesen WO (1995) Neuronal control of leech swimming. J Neurobiol 27:403-418. CrossRef Medline

Chen TW, Wardill TJ, Sun Y, Pulver SR, Renninger SL, Baohan A, Schreiter ER, Kerr RA, Orger MB, Jayaraman V, Looger LL, Svoboda K, Kim DS (2013) Ultrasensitive fluorescent proteins for imaging neuronal activity. Nature 499:295-300. CrossRef Medline

Couton L, Mauss AS, Yunusov T, Diegelmann S, Evers JF, Landgraf M (2015) Development of connectivity in a motoneuronal network in Drosophila larvae. Curr Biol 25:568-576. CrossRef Medline 
Curtin KD, Zhang Z, Wyman RJ (2002) Gap junction proteins are not interchangeable in development of neural function in the Drosophila visual system. J Cell Sci 115(Pt 17):3379-3388. Medline

Davies A, Louis M, Webb B (2015) A model of Drosophila larva chemotaxis. PLoS Comput Biol 11:e1004606. CrossRef Medline

Fox LE, Soll DR, Wu CF (2006) Coordination and modulation of locomotion pattern generators in Drosophila larvae: effects of altered biogenic amine levels by the tyramine beta hydroxlyase mutation. J Neurosci 26: 1486-1498. CrossRef Medline

Fujioka M, Lear BC, Landgraf M, Yusibova GL, Zhou J, Riley KM, Patel NH, Jaynes JB (2003) Even-skipped, acting as a repressor, regulates axonal projections in Drosophila. Development 130:5385-5400. CrossRef Medline

Fushiki A, Zwart MF, Kohsaka H, Fetter RD, Cardona A, Nose A (2016) A circuit mechanism for the propagation of waves of muscle contraction in Drosophila. Elife 5:e13253. Medline

Gjorgjieva J, Berni J, Evers JF, Eglen SJ (2013) Neural circuits for peristaltic wave propagation in crawling Drosophila larvae: analysis and modeling. Front Comput Neurosci 7:24. Medline

Grillner S (2003) The motor infrastructure: from ion channels to neuronal networks. Nat Rev Neurosci 4:573-586. Medline

Heckscher ES, Lockery SR, Doe CQ (2012) Characterization of Drosophila larval crawling at the level of organism, segment, and somatic body wall musculature. J Neurosci 32:12460-12471. CrossRef Medline

Heckscher ES, Zarin AA, Faumont S, Clark MQ, Manning L, Fushiki A, Schneider-Mizell CM, Fetter RD, Truman JW, Zwart MF, Landgraf M, Cardona A, Lockery SR, Doe CQ (2015) Even-skipped ${ }^{+}$interneurons are core components of a sensorimotor circuit that maintains left-right symmetric muscle contraction amplitude. Neuron 88:314-329. CrossRef Medline

Hernandez-Nunez L, Belina J, Klein M, Si G, Claus L, Carlson JR, Samuel AD (2015) Reverse-correlation analysis of navigation dynamics in Drosophila larva using optogenetics. Elife 4:e06225. CrossRef

Inada K, Kohsaka H, Takasu E, Matsunaga T, Nose A (2011) Optical dissection of neural circuits responsible for Drosophila larval locomotion with halorhodopsin. PLoS One 6:e29019. CrossRef Medline

Itakura Y, Kohsaka H, Ohyama T, Zlatic M, Pulver SR, Nose A (2015) Identification of inhibitory premotor interneurons activated at a late phase in a motor cycle during Drosophila larval locomotion. PLoS One 10: e0136660. CrossRef Medline

Juszczak GR, Swiergiel AH (2009) Properties of gap junction blockers and their behavioural, cognitive and electrophysiological effects: animal and human studies. Prog Neuropsychopharmacol Biol Psychiatry 33:181198. CrossRef Medline

Kim MD, Wen Y, Jan YN (2009) Patterning and organization of motor neuron dendrites in the Drosophila larva. Dev Biol 336:213-221. CrossRef Medline

Koh YH, Popova E, Thomas U, Griffith LC, Budnik V (1999) Regulation of DLG localization at synapses by CaMKII-dependent phosphorylation 98:353-363.

Kohsaka H, Okusawa S, Itakura Y, Fushiki A, Nose A (2012) Development of larval motor circuits in Drosophila. Dev Growth Differ 54:408-419. CrossRef Medline

Kohsaka H, Takasu E, Morimoto T, Nose A (2014) A group of segmental premotor interneurons regulates the speed of axial locomotion in Drosophila larvae. Curr Biol 24:2632-2642. CrossRef Medline

Lai SL, Lee T (2006) Genetic mosaic with dual binary transcriptional systems in Drosophila. Nat Neurosci 9:703-709. CrossRef Medline

Landgraf M, Jeffrey V, Fujioka M, Jaynes JB, Bate M (2003) Embryonic origins of a motor system: motor dendrites form a myotopic map in Drosophila. PLoS Biol 1:E41. CrossRef Medline

Lemon WC, Pulver SR, Höckendorf B, McDole K, Branson K, Freeman J, Keller PJ (2015) Whole-central nervous system functional imaging in larval Drosophila. Nat Commun 6:7924. CrossRef Medline

Li HH, Kroll JR, Lennox SM, Ogundeyi O, Jeter J, Depasquale G, Truman JW (2014) A GAL4 driver resource for developmental and behavioral studies on the larval CNS of Drosophila. Cell Rep 8:897-908. CrossRef Medline

Li WC, Roberts A, Soffe SR (2009) Locomotor rhythm maintenance: electrical coupling among premotor excitatory interneurons in the brainstem and spinal cord of young Xenopus tadpoles. J Physiol 587:1677-1693. CrossRef Medline

Macleod GT, Suster ML, Charlton MP, Atwood HL (2003) Single neuron activity in the Drosophila larval CNS detected with calcium indicators. J Neurosci Methods 127:167-178. CrossRef Medline
Mahr A, Aberle H (2006) The expression pattern of the Drosophila vesicular glutamate transporter: a marker protein for MNs and glutamatergic centers in the brain. Gene Expr Patterns 6:299-309. Medline

Manning AK, Hivert MF, Scott RA, Grimsby JL, Bouatia-Naji N, Chen $\mathrm{H}$, Rybin D, Liu CT, Bielak LF, Prokopenko I, Amin N, Barnes D, Cadby G, Hottenga JJ, Ingelsson E, Jackson AU, Johnson T, Kanoni S, Ladenvall C, Lagou V, et al (2012) A genome-wide approach accounting for body mass index identifies genetic variants influencing fasting glycemic traits and insulin resistance. Nat Genet 44:659-669. Medline

Marder E (1998) Electrical synapses: beyond speed and synchrony to computation. Curr Biol 8:R795-R797. CrossRef Medline

Marder E, Calabrese RL (1996) Principles of rhythmic motor pattern generation. Physiol Rev 76:687-717. Medline

Marley R, Baines RA (2011) Dissection of third-instar Drosophila larvae for electrophysiological recording from neurons. Cold Spring Harb Protoc 2011:pii. Medline

Matsunaga T, Fushiki A, Nose A, Kohsaka H (2013) Optogenetic perturbation of neural activity with laser illumination in semi-intact Drosophila larvae in motion. J Vis Exp 77:e50513.

Moore NJ, Bhumbra GS, Foster JD, Beato M (2015) Synaptic connectivity between Renshaw cells and motoneurons in the recurrent inhibitory circuit of the spinal cord. J Neurosci 35:13673-13686. CrossRef Medline

Mulloney B, Smarandache-Wellmann C (2012) Neurobiology of the crustacean swimmeret system. Prog Neurobiol 96:242-267. CrossRef Medline

Ni JQ, Zhou R, Czech B, Liu LP, Holderbaum L, Yang-Zhou D, Shim HS, Tao R, Handler D, Karpowicz P, Binari R, Booker M, Brennecke J, Perkins LA, Hannon GJ, Perrimon N. Nat Methods (2011) A genome-scale shRNA resource for transgenic RNAi in Drosophila. 8:405-407.

Ohyama T, Schneider-Mizell CM, Fetter RD, Aleman JV, Franconville R, Rivera-Alba M, Mensh BD, Branson KM, Simpson JH, Truman JW, Cardona A, Zlatic M (2015) A multilevel multimodal circuit enhances action selection in Drosophila. Nature 520:633-639. CrossRef Medline

Pereda AE (2014) Electrical synapses and their functional interactions with chemical synapses. Nat Rev Neurosci 15:250-263. CrossRef Medline

Pézier AP, Jezzini SH, Bacon JP, Blagburn JM (2016) Shaking B mediates synaptic coupling between auditory sensory neurons and the giant fiber of Drosophila melanogaster. PLoS One 11:e0152211. CrossRef Medline

Phelan P, Goulding LA, Tam JL, Allen MJ, Dawber RJ, Davies JA, Bacon JP (2008) Molecular mechanism of rectification at identified electrical synapses in the Drosophila giant fiber system. Curr Biol 18:1955-1960. CrossRef Medline

Pulver SR, Bayley TG, Taylor AL, Berni J, Bate M, Hedwig B (2015) Imaging fictive locomotor patterns in larval Drosophila. J Neurophysiol 114:25642577. CrossRef Medline

Rela L, Szczupak L (2004) Gap junctions: their importance for the dynamics of neural circuits. Mol Neurobiol 30:341-357. CrossRef Medline

Sanyal S (2009) Genomic mapping and expression patterns of C380, OK6 and D42 enhancer trap lines in the larval nervous system of Drosophila. Gene Expr Patterns 9:371-380. CrossRef Medline

Schneider-Mizell CM, Gerhard S, Longair M, Kazimiers T, Li F, Zwart MF, Champion A, Midgley FM, Fetter RD, Saalfeld S, Cardona A (2016) Quantitative neuroanatomy for connectomics in Drosophila. Elife 5:pii: e12059. CrossRef Medline

Song J, Ampatzis K, Björnfors ER, El Manira A (2016) Motor neurons control locomotor circuit function retrogradely via gap junctions. Nature 529:399-402. CrossRef Medline

Stebbings LA, Todman MG, Phillips R, Greer CE, Tam J, Phelan P, Jacobs K, Bacon JP, Davies JA (2002) Gap junctions in Drosophila: developmental expression of the entire innexin gene family. Mech Dev 113:197-205. CrossRef Medline

Tastekin I, Riedl J, Schilling-Kurz V, Gomez-Marin A, Truman JW, Louis M (2015) Role of the subesophageal zone in sensorimotor control of orientation in Drosophila larva. Curr Biol 25:1448-1460. CrossRef Medline

Tresch MC, Kiehn O (2000) Motor coordination without action potentials in the mammalian spinal cord. Nat Neurosci 3(6):593-9. CrossRef

Vogelstein JT, Park Y, Ohyama T, Kerr RA, Truman JW, Priebe CE, Zlatic M (2014) Discovery of brainwide neural-behavioral maps via multiscale unsupervised structure learning. Science 344:386-392. CrossRef

Zwart MF, Pulver SR, Truman JW, Fushiki A, Fetter RD, Cardona A, Landgraf M (2016) Selective inhibition mediates the sequential recruitment of motor pools. Neuron 91:944. CrossRef Medline 